




\section{SYSTEILITISCIIES VERZEICIINISS}

$$
\text { der }
$$

\section{VRrsteinerungen DES HeLVPTIN}

DER SCWEIZ UND SCIIWABENS

von

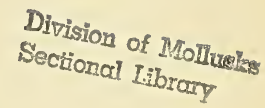

D 1. Ira l M a yen

in Zürich.

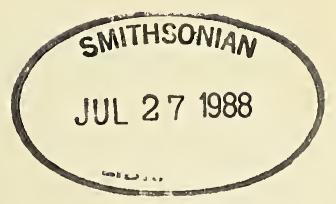

Zürich.

Schabelitz'sche Buchhandlung.

(Cæsar Schmidt.)

1872. 
Meyer'sche Buchdruckerei in Luzern. 


\section{VORWORT.}

Zwei Dezenien sind beinahe verstrichen, seit mein erstes Verzeichniss der Petrefakten der schweizerischen Meeres-Molasse erschienen ist. ${ }^{1}$ Während dieser langen Zeit und wie schon verschiedene, inzwischen veröffentlichte partielle Verzeichnisse ${ }^{2}$ es ahnen liessen, hat sich nach und nach die Zahl der in unserem so ausgedehnten Gebiete des Helvétien aufgefundenen Arten um beinahe das Doppelte vermehrt, ja sie dürfte sich heute, bei gründlicher Bearbeitung des in unseren Musæen und kleineren Sammlungen aufbewahrten Materials, bereits auf nahezu tausend belaufen. Unter diesen Umständen und gegenüber der raschen Publikation unserer übrigen fossilen Faunen und Floren, ist es wohl angezeigt, endlich, in Ermanglung einer für meine bescheidenen Verhältnisse zu kostspieligen Arbeit, nach einem der Sache würdigen Plane, eine neue, vermehrte und verbesserte systematische Aufzählung unserer so reichen und interessanten „miocänen“ marinen Faunen dem Drucke zu übergeben, und ich folge daher dem Wunsche meines Freundês, Professor Kaufmann, indem ich den Anlass der Veröffentlichung seines Textes zu Blatt VIII der schweiz. geologischen Karte

1 Mittheilungen der naturforschenden Gesellschaft in Bern. 1853.

2 Uebersicht der Geologie des Kantons Zürich. (Neujahrsblatt der naturforschenden Gesellschaft in Zürich. 1862.) - Mosch, Geologische Beschreibung des Kantons Aargau. 1867. - Mayer, Catalogue systématique, etc.; Chénopides, Strombides et Ficulides. 1867; Mactrides et Pholadomyides. 1867; Arcides. 1868; Panopéides. 1870. 
(Beiträge zur geolog. Karte der Schweiz, Lief. XI) zu der Einlage eines solchen allgemeinen Verzeichnisses benutze.

Leider ist, in Folge der kurzen Zeit, welche mir zur Revision und zur Erweiterung des Rahmens meines alten Katalogs gegeben worden, gegenwärtige Arbeit nicht so vollständig und verhältnissmässig vollkommen geworden, als sie es, unter günstigeren Umständen hätte werden können. Namentlich thut es mir Leid, nicht Zeit gehabt zu haben, die zahlreichen, kleinen Bivalven der Luzerner und Beruer Meeres-Molasse und die noch zahlreicheren und besser erhaltenen, kleinen Gastropoden des Jura-Grobkalkes und des Muschelsandsteines alle gründlich zu studieren, denn ich zweifle nicht daran, dass ich so, nur aus dem auf den Zürcher Sammlungen befindlichen Materiale, an hundert Arten mehr anzuführen und, andrerseits, eine Anzahl irrthümlicher Bestimmungen zu verbessern Gelegenheit gehabt hätte. Indessen wird immerhin ein vergleichender Blick auf gegenwärtige und auf die Arbeit von 1853 einen schönen Fortschritt in der Arten-Zunahme, in der Terminologie und in der Feststellung der geologischen und paläontologischen Daten bekunden und so, ich hoffe es, das Mangelhafte vor dem Dargebotenen vergessen lassen.

Die Eintheilung meines Verzeichnisses habe ich auf acht Columnen, statt auf sechszehn, welche die Berücksichtigung aller Stufen, in welchen Einzelne unserer Arten vorkommen, verlangt hätte, beschränkt, in der Meinung, dass solche umständliche Anführungen in einer Arbeit mit speziellem Zwecke nicht am besten Platze seien. Ja, ich hätte füglich die vorletzte, das Messinian und das Astian vertretende Columne auslassen können, ohne meinem Hauptzwecke, einen Einblick in die Vertheilung der Arten der Molasse ausserhalb ihrer Grenzen zu erlauben, grossen Schaden anzuthun; allein ich wollte schliesslich die gute Gelegenheit nicht unbenutzt lassen, durch die Angaben über das Hinaufgehen so vieler unserer Arten bis in die genannten zwei obersttertiären Stufen, wieder ein Mal zum Beweise beizutragen, dass die alte Unterscheidung von Miocæn und Pliocæn rein willkürlich und unstatthaft sei.

In Betreff des hier befolgten geologischen Schema's, kann ich wohl, An- 
gesichts der in meinen Schriften ${ }^{1}$ und der in neuerer Zeit von den östreichischen Geologen ${ }^{2}$ endlich festgestellten Thatsachen, mich kurz fassen und statt ausführlicher Darlegungen, mehr eine dem Gedächtniss zu Hülfe kommende Aufzählung der Unterabtheilungen und ihrer Hauptablagerungen geben, welche bei gegenwärtiger Arbeit in Betracht kommen. Hier nun diese Uebersicht.

Vor Allem brauche ich nunmehr kaum zu wiederholen, dass die TertiärStufe, welche ich, anno 155\%, mit Recht Helvetian genannt habe, da ihre Ablagerungen nirgends, ausser im Wiener Beckell, so vollständig und grossartig entwickelt sind, wie auf schweizer Gebiet, zu den natürlichsten, das heisst, in geographischer, stratigraphischer und paläontologischer Beziehung best begrenzten Stufen gehört und dass sie, speziell in der Schweiz, in dieser Rücksicht Nichts zu wünschen übrig lässt. Ebenso fest steht es heute, dass diese Stufe in drei, durch ihre Fannen sowohl als durch die Gesteinsbeschaffenheit merklich von einander abweichende Unterabtheilungen zerfällt, für welche ich die Namen: Grunder-, Serravaller- und St. GallerSchichten vorgeschlagen habe. Was nun die Haupt-Ablagerungen jedes dieser Niveaux betrifft, so sind es, in Europa, folgende: Die untere Abtheilung des Helvetian besteht, erstens, aus einem mehrfach unterbrochenen, langen Streifen Meeresniederschläge, der aus der Gegend von Bordeaux (Gabarret, Sos, Reimbez) über Poitiers (Nirebeau) nach der Tourraine und bis Moulins reicht; dann, im Jura, vom Departement gleichen Namens, über Court, den Mettenberg und die Plateanx von Baselland und des Aargau's, nach dem Randen und bis Bachzimmern und Winterlingen, an der würtembergischen Donau, sich erstreckt; ferner in der Mitte des Wienerbeckens sich wiederfindet und, wahrscheinlich über Galizien, nach Volhynien hinübergeht. Zweitens aber ist diese untere Abtheilung längs des Nordfusses des ligurischen Apennins und in der Superga-Kette bei Turin (hier

1 Mayer, locis citatis; Tableau synchronistique des terrains tertiaires supérieurs. 1868; Décourerte des couches à Congéries dans le bassin du Rhône. 1871 .

2 Jahrbuch der k. k. östreich. geolog. Reichs-Anstalt, 1871, pag. 327. 
sehr Petrefakten-reich) entwickelt, während sie, drittens, wahrscheinlich auch in Südfrankreich (zu le Sausset bei les Martigues), wenn auch schlecht entwickelt, vorhanden ist.

Die mittlere Abtheilung, fast n̈berall gekennzeichnet durch ihre GesteinsBeschaffenheit, als gelblicher Molasse-Sandstein, und durch eine Menge von Bryozoen, von Echinodermen und von Haifischzähnen, folgt, mit orographischstratigraphischer Nothwendigkeit, auf die erste in der Gegend von Gabarret und Sos; ebenso im Loire-Thal, bei Savigné nördlich von Tours; ebenso im Jura (am Randen) und, in ganz prägnanter Weise, in der Superga-Kette und bei Serravalle-di-Scrivia; während sie, paläontologisch unverkemnbar, bei Montpellier (Juvignac etc.) wieder auftritt und hier der typisch entwickelten dritten Abtheilung deutlich als Basis dient.

Die obere Abtheilung endlich, ebenfalls auf weiten Strecken in ihren paläontologischen und petrographischen Charakteren constant (ich erinnere an die blauen oder gelben Mergeln mit Turritellen-, mit Tapes - und mit PanopæenSchichten, von Montpellier, von St. Mitre bei les Martigues, von Bern, Luzern, St. Gallen, von Trento, von Salles etc., und an den Nulliporenoder Leitha-Kalk von ganz Südfrankreich, von Serravalle-di-Scrivia, der Umgegend von Wien etc.), - diese obere Abtheilung überlagert die mittlere, orographisch sicher, bei Bordeaux (Saucats-Salles) und. sichtbar, bei Montpellier, bei Luzern (Profil Löwendenkmal-Rothsee) bei St. Gallen (Martinsbrücke, Staad), bei Turin (Pino) und bei Serravalle (am Ufer der Scrivia). Es ist daher an ihrer Selbstständigkeit als eigene Unter-Abtheilung nicht im Mindesten zu zweifeln.

Dass die auf das Helvetian folgende Stufe, das Tortonian, wirklich eine eigentliche Stufe und nicht bloss eine weitere Unter-Abtheilung des Helvetian sei, lässt sich heute sehr leicht, sowohl auf stratigraphischem als auf paläontologischem Wege nachweisen. Vor Allem ist es nunmehr wohl allgenein anerkannt, dass diese Stufe unmittelbar auf das obere Helvetian folgt (so im klassischen Profile von Serravalle nnd so, nach endlicher Festsetzung durch die Wiener Geologen, zu Baden bei Wien). Was nun ihre Facies betrifft, so ist dieselbe bekanntlich ebenso durch die Gesteinsbeschaf- 
fenheit (ihre marinen Ablagerungen scheinen überall aus blauen Thonen zu bestehen), als durch die Fauna, welche, bereits und plötzlich, an diejenige des unteren und mittleren Astian erinnert, ganz ausgezeichnet und kaum mit derjenigen einer andern Abtheilung zu verwechseln. Kurz, die Stufe unterscheidet sich, in paläontologischer Beziehung, viel mehr vom oberen Helvetian als dieses vom mittleren oder diese mittlere Abtheilung von der unteren. Ebenso unabhängig aber ist die eilfte Tertiärstufe in stratigraphischer oder geographischer Beziehung. Beweise sind eines Theiles, ihr Auftreten in neu gestaltete, schmale und tiefe Becken, wie zwischen Dax und Bayonne, in der Nähe und südlich von Wien, zu Lapugy in Siebenbürgen, bei Messina, in Caramanien etc., andern Theils, ihr Ersatz von Meeres-Niederschläge durch Süsswasser-Bildungen, wie im Jura und in der Ostschweiz.

Es lohnt sich kaum der Mühe, hier ein Wort über die stratigraphischen Verhältnisse der auf das Tortonian folgenden Stufe, des Messinian, zu verlieren, da dieselben bereits in zahlreichen Schriften beleuchtet und heute wohl allgemein bekannt sein dürften. Für diejenigen, welche mit der Litteratur über die Tertiär-Formation weniger vertraut sind, sei indessen.gesagt, dass die zwölfte T'ertiär-Stufe, ein Mal, als rein marine Ablagerung, bei Messina, regelmässig auf die Eilfte folgt; als mehr oder weniger brackische Bildung, bei Tortona (Serravalle, S. Agata), sowie bei Wien, jene eilfte Stufe unmittelbar überlagert; dann aber, bei Bollène in Südfrankreich sowohl, als in ganz Ungarn und in ganz Südrussland, unabhändig vom Tortonian auftritt und auch so ihre Selbstständigkeit als Stufe bekundet.

Das Astian seinerseits (die einzige Stufe, die von Anfang an richtig aufgefasst und - als pliocæn - in ihrer jetzigen Begrenzung festgestellt wurde), überlagert das Messinian an vielen Stellen Italiens, so bei Messina, bei Tortona (Villalvernia), bei Tabbiano etc.; es ist aber in weitaus den meisten Gegenden, wo es auftritt, von den älteren Stufen vollkommen unabhängig, wodurch wieder bewiesen wird, dass es einer eigenen Epoche entspricht.

Nach diesen wohl genügenden Bemerkungen über die geologischen Thatsachen, welche dem in meinem Verzeichniss befolgten Schema zu Grunde 
liegen, erübrigt mir noch, spezieller über die Resultate zu berichten, welche die Vergleichung der hier unterschiedenen Faunen mit denjenigen des ausländischen Helvetian und auch der zunächst folgenden Stufen, an der Hand meines Verzeichnisses liefert, mit andern Worten, die eben angeführten, auf der durch die Orographie, die Petrographie nnd die Facies der Faunen unterstützten Stratigraphie beruhenden Unterscheidungen und Assimilationen durch die Paläontologie zu controlliren und sie durch diese Bestätigungsweise auf den Rang von unumstösslichen Thatsachen zu heben. Diese Controllirung lässt sich auf wenigstens zweierlei Arten bewerkstelligen, nämlich: ein mal, auf statistischem Wege, durch Zusammenzählen der Arten, welche in jeder Columne angeführt werden (wobei zwei fragliche Vorkommen für eine Art gezählt werden mögen) und Vergleichung der so erhaltenen Summen; dann, auf dem Wege des Darwinismus, durch Zuratheziehen der Grösse-, Individuen-Zahl und Stammbaum-Entwicklung der häufigsten oder bekanntesten Arten und Arten-Gruppen in den verschiedenen Niveaux, wo sie auftreten. Bei der ersten Methode bieten sich uns folgende Momente dar: Wir vergleichen, erstens, die Gesammtsumme der Arten des schweizerischen Helvetian mit derjenigen der gleichen Arten in den benachbarten Columnen; zweitens, die Zahl der Arten unseres unteren Helvetian mit derjenigen der gleichen Arten, welche, a) in den älteren Stufen, b) im Helvetian I des Auslandes etc., vorkommen; drittens die Summe der Arten unseres mittleren Helvetian anf die gleiche Weise, und viertens, ebenso die Arten des oberen Helvetian der Schweiz. Gehen wir denn an diese Vergleichung.

In Betreff der ersten Frage, erweist es sich, dass von unseren $\% 40$ schweizerischen Arten, nur $3 \% 1$ oder $50 \%$ schon im Langhian oder früher aufgetreten sind; dass aber 531 oder fast $\left.72^{\circ}\right|_{0}$, oder nach Abzug der 120 nur aus der Schweiz bekannten Arten ( $\% 40$ weniger 120 gleich 620 ) fast $\left.90^{\circ}\right|_{0}$ auch im ausländischen Helvetian vorkommen; dass, ferner, nur 394 oder $53{ }^{\circ} \%_{0}$ in's Tortonian, nur noch 345 oder $42^{\circ} \%_{0}$ in's Messinian und Astian hinaufgehen; endlich, bloss 219 oder nicht ganz $30^{\circ} \%_{0}$ noch leben. Damit wäre denn bereits eine erste Bestätigung der Richtigkeit unser geologischen Reihen- 
folge gefunden. Schauen wir jetzt nach, wie sich die Lösung der zweiten Fragen-Reihe in dieser Beziehung verhält.

Hier zeigt es sich, dass von unseren 141 Species aus dem unteren Helvetian, 88 oder $\left.62{ }^{\circ}\right|_{0}$ aus älteren Stufen stammen (also $12{ }^{\circ} \%_{0}$ mehr als solcher Arten im schweizerischen Helvetian im Allgemeinen vorhanden sind); dass 121 Species aber, oder $\left.86{ }^{\circ}\right|_{0}$, im ausländischen Helvetian I, gegen nur 96 oder $\left.68^{\circ}\right|_{0}$ im Helvetian II, nur noch 93 oder $\left.65^{\circ}\right|_{0}$ im Arten-reichen Helvetian III des Auslandes und noch 91 oder $\left.64^{\circ}\right|_{0}$ im ebenfalls wohlerforschten Tortonian vorkommen, während $\% 1$ Arten oder $50{ }_{0}^{\circ}$ bis in's Messinian oder Astian und 50 Arten oder $35{ }^{\circ}$ in die Jetztwelt hinaufreichen. Die stärkste Quote von gemeinsamen Arten fällt daher auf das untere Helvetian und stimmt also mit unserer Klassifikation überein.

Wir haben ferner aus dem Helvetian II der Schweiz 400 Arten verzeichnet; von diesen stammen nur 233 oder $\left.55{ }^{\circ}\right|_{0}$ aus älteren Stufen, 300 aber oder $\left.75^{\circ}\right|_{0}$ aus dem Helvetian I Europa's; 258 oder $64^{\circ} \%_{0}$ finden sich im ausländischen Helvetian II, trotzdem dass diese Unterabtheilung theils Arten-arm, theils schlecht erforscht ist; ferner gehen 245 Arten oder $61^{\circ}{ }_{0}$ davon in's obere Helvetian; 228 oder $5 \% \%_{0}$ in's Tortinian; 200 oder $50 \%$ in's Messinian und Astian hinauf, während noch 123 oder $\left.30^{\circ}\right|_{0}$ recent vorkommen. Aus diesen Vergleichen erhellt einerseits die grosse paläontologische Verwandtschaft unseres mittleren Helvetian mit dem Unteren des Auslandes (hauptsächlich des Loire-Beckens) und anderseits seine grössere Uebereinstimmung in der gleichen Hinsicht mit dem mittleren Helvetian als mit dem Oberen, trotz der grossen Verbreitung und dem Arten-Reichthum Dieses und es wird durch beide Daten die Richtigkeit unserer Klassifikation . bestätigt.

Das schweizerische obere Helvetian endlich hat uns, laut Verzeichniss, bis jetzt 496 Arten geliefert. 256 oder nur $\left.51{ }^{0}\right|_{0}$ von diesen kommen schon im Langhian oder tiefer vor (gegen $\left.62{ }^{\circ}\right|_{0}$ der Arten unseres unteren und $55^{0}{ }_{0}$ derjenigen unseres mittleren Helvetian); 333 oder $6 \%{ }_{0}$ schon finden sich im ausländischen Helvetian I; 300 oder $\left.60^{\circ}\right|_{0}$ im mittleren; ebensoviel im oberen Helvetian, und es gehen 336 Arten oder $6 \%^{\circ} \%_{0}$ in's Tortonian; 
$25 \mathrm{~S}$ oder $52{ }^{\circ} \%_{0}$ in's Messinian und Astian und 160 oder $32{ }_{0}$ in die Jetztwelt hinanf.

Aus diesen statistischen Angaben geht, klar und bestimmt, die Bestätigung der festgestellten Reihenfolge unserer drei Niveaux des Helvetian hervor, indem sie, mit aller wünscharen Genauigkeit, das Steigen und Fallen der Procente der gemeinsamen Arten der aufgestellten stratigraphischen Norm folgen lassen. Dass aber die Uebereinstimmung der statistischen Resultate mit den stratigraphischen auch hier, trotz der Unvollständigkeit der jenen zu Grunde liegenden Daten und trotz der, natïrlich, sehr grossen Faunen-Uebereinstimmung der verschiedenen Unterabtheilungen einer und derselben Stufe, eine so vollständige ist, beweist wieder ein Mal welche, man möchte sagen wunderbare Macht der Statistik innewohnt, auch unter den scheinbar ungünstigsten Verhältnissen die Wahrheit hervortreten zu lassen.

Indessen und zum Ueberflusse bietet sich uns noch ein der Paläontologie entnommenes und ebenso sicheres Mittel als die Statistik dar um die Reihenfolge unserer drei Niveaux des Helvetian festzustellen. Es ist diess, wie gesagt, die Verfolgung der Verbreitungsweise gewisser Arten und des Stammbaumes gewisser Arten-Gruppen in diesen verschiedenen Niveaux und wenn nöthig ausserhalb Dieser. Aus der Fülle der in dieser Beziehung mir vorliegenden Daten einige prägnante und leicht controlirbare Fälle hervorzugreifen, wird; bei dem absoluten Fehlen von entgegengesetzten Thatsachen, hier wohl genügen, um unser Beweis-Verfahren zu vervollständigen und endgültig die Frage nach dem respektiven Alter unserer drei SchichtenSysteme des Helvetian abzuschliessen. Hier denn einige solche Thatsachen.

1. Bekanntlich finden sich die zwei weitverbreiteten und leichtkenntlichen Arca-Arten, A. Breisbacki und A. Turonica, massenhaft im unteren Helvetian sowobl der Touraine als von Grund bei Wien, worin sie aus dem Langhian oder dem Aquitanian hinaufreichen. Nun ist Erstere im Helvetian II der Schweiz (Niederhasli, Würenlos, Othmarsingen) in den beiden Tourainer Varietäten, die flache und die aufgedunsene, nicht selten, während sich, meines Wissens, noch kein Exemplar der Art im Helvetian III gefunden 


\section{$-9-$}

hat. Ebenso kommt die zweite Art massenhaft wie im Helvetian I und II des Loire-Thales, im Muschelsandstein vom Kalofen bei Brugg, und nicht selten bei Ulm vor, während sie unserem Helvetian III vollständig fehlt und auch im Ausland (Salles) in diesen Niveau schon selten ist. Umgekehrt fehlt die erst im Helvetian I von Turin auftretende jüngere Art, A. diluvii, noch unserem mittleren Helvetian, nicht aber unserem Helvetian III. Schon diese Verhältnisse, zu denen sich das Verhalten der weiteren Arca-Arten, A. Fichteli, A. Helvetica, A. scabrosa, hinzugesellt, deuten deutlich darauf, dass der Muschelsandstein dem unteren Helvetian chronologisch näher steht als die sulbalpine Meeres-Molasse.

2. Zu den lehrreichsten Thatsachen im Gebiete des auf die Stratigraphie angewanten Lehre von der: natürlichen Entstehung der Arten durch Variabilität und Racenbildung gehört das Verhalten der zwei bekannten CardienGruppen, der Gruppen des C. echinatum und des C. edule, in den drei Niveaux des Helvetian. Die ersten Verwandten des C. echinatum in Aquitanian und Langhian, C. Girondicum und C. Leognanicum, sind bekanntlich ziemlich kleine Formen. Schon etwas grösser wird C. Saucatsense, aus dem oberen Langhian, dessen Entwicklung aus C. Girondicum mir an der Hand meiner Individuen-Serien nachzuweisen möglich ist. Noch grösser wird C. hispidum, aus dem Helvetian I von Volhynien, welche Art mit ihrem unmittelbaren Vorgänger C. Saucatsense zunächst verwandt ist. Im Helvetian I der Touraine (und auch wohl von Turin und Wien) nun sind die Formen unserer Gruppe, C. Turonicum, C. Andreæ, C. Dujardini, C. Michelottii, noch klein, wie die früheren; dafür ähnelt bereits Jede mehr oder weniger einem späteren Typus; C. Andreæ dem C. erinaceum; C. Dujardini dem C. echinatum; C. Michelottii dem C. aculeatum. Nun finden sich dièse ersten Typen unverändert und nicht sehr selten noch im Helvetian II der Schweiz, neben seltenen Individuen der recenteren und grösseren Formen, C. aculeatum, C. erinaceum, C. tuberculatum (verwandt mit C. Saucatsense), während sie dem Helvetian III vollständig fehlen und hier nur die grösseren Arten, C. hispidum, C. echinatum vorkommen. Diese Thatsachen deuten ihrerseits wieder auf die nahe geologische Verwandtschaft unseres Muschel- 
sandsteines mit dem unteren Helvetian und auf die grössere chronologische Entfernung der Meeres-Molasse von letzterem Horizonte.

Ganz ähnlich verhalten sich in der Schweiz die Arten aus der Gruppe des C. edule. Die ersten hieher gehörigen Formen, C. Lucernense, aus dem Aquitanian, C. sociale, aus dem Langhian? etc., sind ganz klein. Wenig grösser sind die folgenden C. rotundatum und C. arcella aus dem Helvetian I. Etwas grösser, doch nie so gross wie alte Individuen des C. edule, wird das meist hohe und bauchige, mit nur 20 bis 22 hohen, tief abgetrennten und stark gekerbten Rippen versehene C. commune, das aus dem Langhian und Helvetian I von Wien so massenhaft in's Helvetian II der Schweiz hinübergeht. Dieses nun wird in unserem Helvetian III durch das C. lapicidinum ersetzt, welches sich durch seine quere Form und etwas zahlreicheren Rippen noch näher dem C. edule anlehnt. Während ferner das recente C. crassum im Muschelsandsteine noch eine Seltenheit ist, wird es in der Meeres-Molasse bei Belp und vom Burgerwald bei Freiburg schon ziemlich häufig. Während das grosse C. præcellens, das gewissen Varietäten des C. edule von Alexandria sich anschliesst, im Helvetian II nur im Hohgau nicht selten ist, kommt es in der Molasse von St. Gallen zu seiner vollen Grösse- und Zahl-Entwichlung. Es sind dies aber lauter kleine Daten, welche in der gleichen Weise, das heisst für das höhere Alter des Muschelsandsteines als der Meeres-Molasse, zeugen.

Ich schliesse mit der Vorführung des in geologischer wie in stratigraphischer Beziehung gleich lehrreichen Entwicklung der kleinen aber gut abgeschlossenen und an der Kerbung der Seitenzähne etc. leicht kenntlichen Gruppe der Mactra triangula, welche eine nicht unwichtige Rolle in der hier abzuschliessenden Debatte spielt. Mactra triangula, bekanntlich schon häufig, normal und wenig veränderlich im unteren Langhian vom Moulin de l'Eglise, zu Saucats, wird im Helvetian I der Touraine zu der allergemeinsten Muschel und fängt zur gleichen Zeit, nach drei Richtungen hin, $\mathrm{zu}$ varïiren an. Untersucht man nämlich z. B. 1000 Individuen oder Klappen von ihr, so findet man, dass kaum 400 davon normal sind, d. h. ein queres und gleichschenkeliges Dreieck bilden, während circa 100 Stücke 
allmählig kürzer, höher und etwas bauchiger, 200 länger (breiter) und flacher und die Uebrigen ungleichseitig werden. Zugleich findet man, auf die Menge Stücke, einige wenige, welche das normale Mass (12 Millimeter) um bis 5 Millimeter überschreiten und daher, wenigstens die grössten Individuen, bereits, die Kurzen, als M. solida, die Breiten, als M. ovalis und die Ungleichseitigen, als M. subtruncata gelten müssen. Nun erweist es sich, dass die typische M. triangula im Helvetian II der Schweiz ebenfalls massenhaft vorkömmt; dass sie hier aber durchschnittlich um ein Paar Millimeter grösser wird als im Helvetian I und dass neben ihr, nicht selten, Exemplare von M. solida, M. ovalis und M. subtruncata auftreten, welche in ihren Dimensionen die Mitte zwischen den grössten Stücken aus dem Helvetian I und den ausgewachsenen Individuen aus dem Crag oder den europäischen Meeren einhalten. Dass nun ein natürlicher Zusammenhang zwischen diesen vier Formen, d. h. die Entstehung von M. solida, M. ovalis und M. subtruncata aus M. triangula, im Helvetian I der Touraine und hingegen bereits eine scharfe Abtrennung der gleichen Arten im Helvetian II der Schweiz vorliegt, ist wohl nicht zu läugnen und ebensowenig, dass die gleichmässige Vertheilung der Arten-Gruppe in den Faluus des Loire-Beckens und im Muschelsandsteine der Schweiz auf den innigen chronologischen Anschluss beider Bildungen hindeutet. Das Fehlen der im europäischen Ocean so gemeinen M. solida, ovalis und subtruncata in der schweizerischen Meeres-Molasse, seiner Seits, möchte wohl nur im ersten Momente auffallen und theils mit dem für diese Sandbewohner zu schlammigen Grunde des Molasse-Meeres, theils mit der durch hundert tropische Formen bewiesenen, auf's Neue wärıneren Facies der Meeres-Molasse genügend erklärt sein. 


\section{VIRERTIOHNISS}

der

\section{Versteinerungen des HELVETIAN der Schweiz und Schwabens.}

NT3. 1 bedeutet: sehr selten: 2, selten; 3, weder selten noclı häufig; 4, häufig, und 5 , sehr hăufig. - $\mathbf{N}$ bezeichnet. die Nordzone, s die Südzone, Luz die Zone Luzern-(Rothsee)-St. Gallen, Be die Schichten südwärts von Bern.

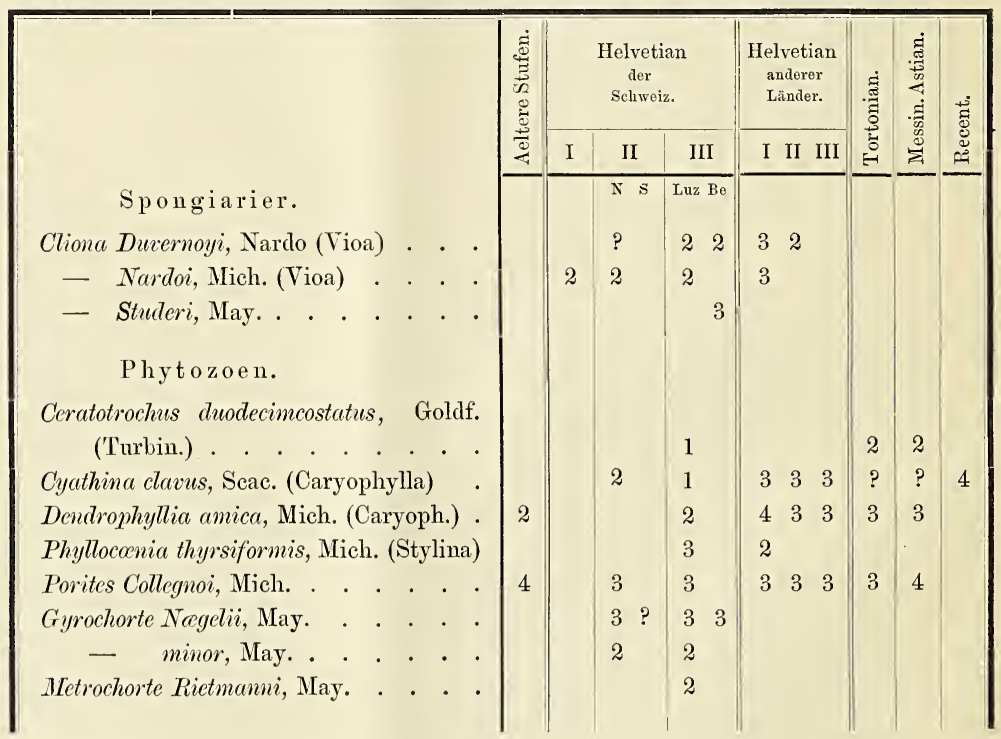




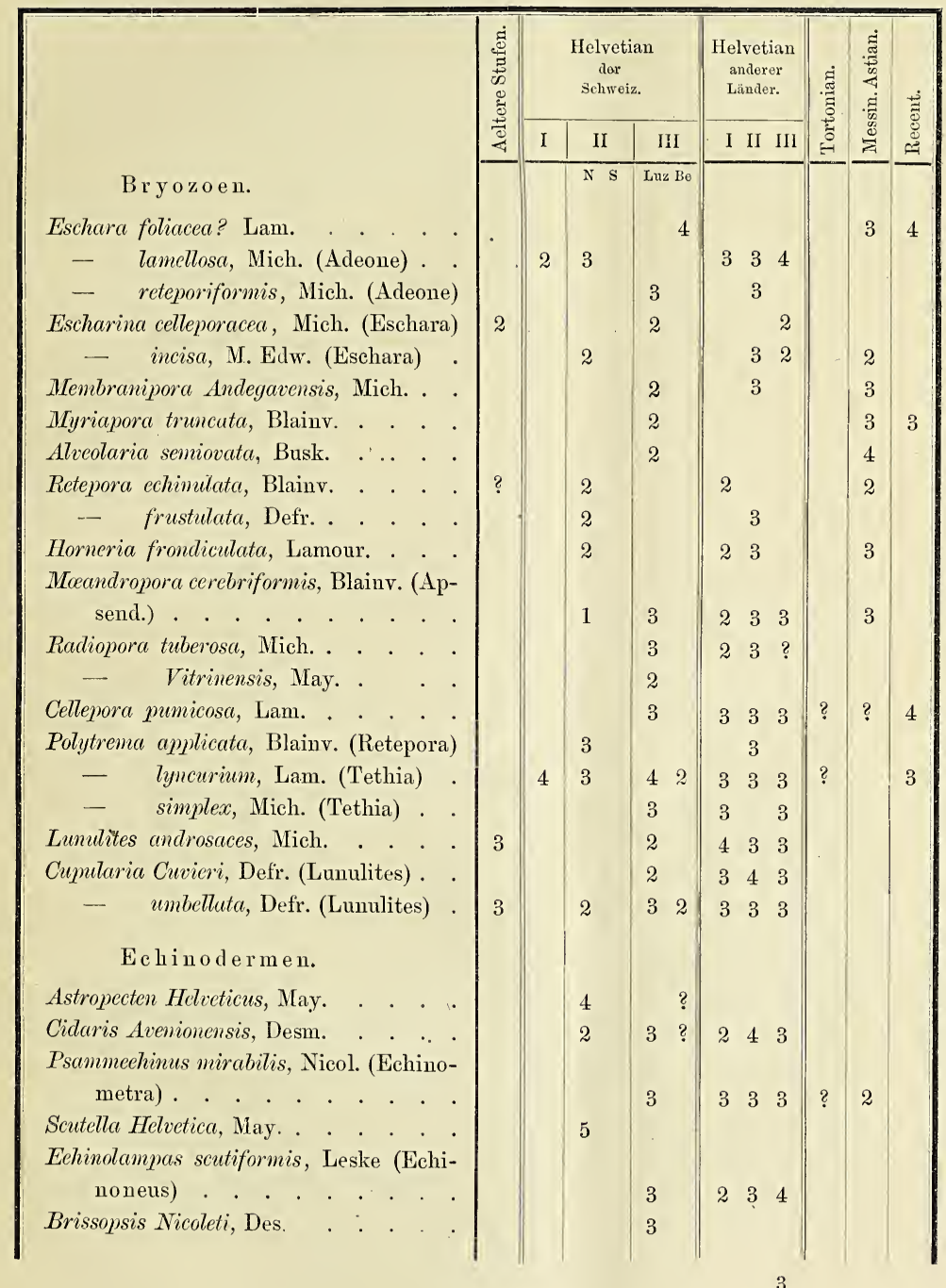


Schizaster Scillai, Ag. . . . . . .

Echinocardium Dcickei, Des. . . . .

Brachiopoden.

Lingula ovalina, May.

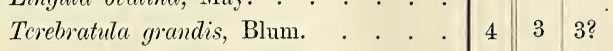

- Harncsi, Suess. . . . . 4

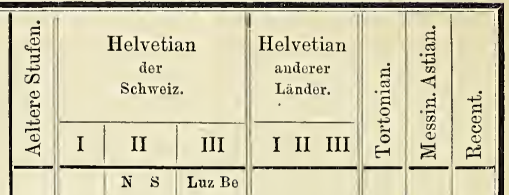

- mioconica, Mich. . . . .

(Anomia)

Pelecypoden.

Anomia ephippium, L. . . . . . . Ostrea (Gryphea) cochlear, Poli.. . .

- Addolii, May.

- arcnicola? May. .

- Argoriana, May. .

- batillum, May.

- Boblayei, Desh. . . . . . .

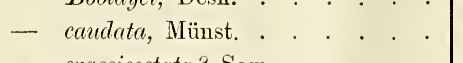

- crassicostata? Sow. . . . . .

- crassissima, Lam. . . . . . 2

- cucullata, Born. . . . . . . .

- cdulis, L. . . . . . . . . . .

- emarginata, Münst. . . . . .

- foveolata, Eichw.

- Helvetica, May. . . . . . .

- hyotis, L. (Mytilus) . . . . .

- lacerata, Goldf. . . . . . .

- lamellosa, Broc. . . . . . . 3

- Mcriani, May.

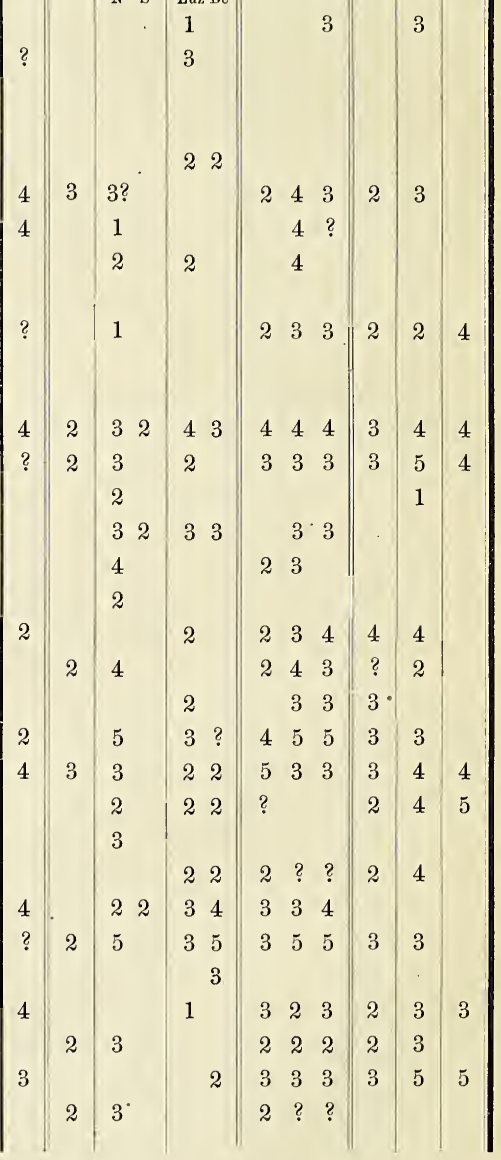




\begin{tabular}{|c|c|c|c|c|c|c|c|c|c|c|c|c|}
\hline \multirow{4}{*}{\multicolumn{3}{|c|}{$\begin{array}{l}\text { Ostrea mixta, May. } \\
\text { - Moschi? } \text { May. } \\
\end{array}$}} & 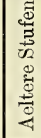 & \multicolumn{3}{|c|}{$\begin{array}{c}\text { Helvetian } \\
\text { der } \\
\text { Schweiz. }\end{array}$} & \multicolumn{3}{|c|}{$\begin{array}{c}\text { IIelvetian } \\
\text { anderer } \\
\text { Länder. }\end{array}$} & 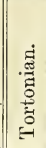 & 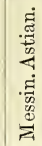 & 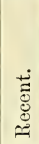 \\
\hline & & & & & N $\mathrm{s}$ & Luz Bo & & & & & & \\
\hline & & & & & 4 & & & & & & & \\
\hline & & & & & 1 & & & & & & & \\
\hline - neglecta, Mich. . . . . & & . & 5 & & & 11 & & 3 & & ? & & \\
\hline - tegulata, Münst. . . . & & . & & & 3 & 23 & & 2 & & 2 & & \\
\hline - Virginiana, Gm. . . . & & . & 4 & 3 & 4 & & 5 & 4 & & 4 & $?$ & 5 \\
\hline Plicatula mytilina, Phil. . . & & . & 3 & & & 2 & & 3 & & 4 & 3 & ? \\
\hline - ruperella, Duj. . . & & 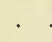 & 3 & ? & & 1 & & 3 & & 3 & & \\
\hline Himites Brussoni, Serr. . . & & . & & & 2 & 3 & & 2 & & 2 & 2 & \\
\hline Pecten (Neithea) benedictus, Lam & & $\cdot$ & & 2 & 42 & 23 & & 4 & 4 & 4 & & ? \\
\hline Beudanti, Bast & & . & 4 & & & 2 & & 2 & 2 & 2 & & \\
\hline gigas, Schl. . & & . & 4 & & $? 4$ & & $?$ & $?$ & & & & \\
\hline - Hermannseni, & & unk. & $?$ & & 4. 3 & 54 & 3 & 4 & 5 & & & \\
\hline latissim., Broc. & & trea) & & & & 2 & & 4 & 4 & 3 & 3 & \\
\hline Marice, May. & & 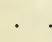 & & & 2 & & & & & & & \\
\hline - solarium, Lam. & & . & & & & 23 & 3 & 4 & 4 & $?$ & & \\
\hline - Celestini, May. . . . & & . & & & & 2 & & & & & & \\
\hline - Islandicus? Müll. (Ostrea & & 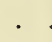 & & & 1 & & 2 & $?$ & ? & & & 4 \\
\hline - Jaccardi, May. . . . & & 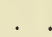 & & & & 2 & & & & & & \\
\hline - laviplex, May. . . . & & . & & & 2 & & & & & & & \\
\hline - opercularis, L. (Ostrea) & & $\cdot$ & 5 & & 3 & 3 & 5 & 5 & 5 & 4 & 5 & 5 \\
\hline - palmatus, Lam. . . . & & . . & $?$ & & 44 & 44 & & 3 & 3 & & & \\
\hline - Probsti, May. . . . . & & . & & & 1 & & & & & & & \\
\hline - pusio, L (Ostrea) . . & & . & 3 & 3 & 32 & 42 & 4 & 4 & 4 & 4 & 4 & 5 \\
\hline - Puymorice, May. . . . & & . & & & 2 & & 3 & 4 & & & & \\
\hline - scabrellus, Lam. . . . & & . & 2 & 2 & 32 & 43 & 3 & 44 & 4 & 4 & 5 & \\
\hline - scabriusculus? Math. . & & 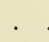 & & & & 2 & & & & 3 & & \\
\hline - Turiccnsis, May.. . . & & • & & & 1 & & & & & & & \\
\hline - vcntilabrum, Goldf. . . & & . & & 2 & 4 & 3 & & 53 & & & & \\
\hline Lima Corrodii, May. . . . & & . & & & 3 & & & & & & & \\
\hline - hians, Gm. (Ostrea). . & & . & 3 & & 2 & 2 & 2 & 2 & & 2 & 3 & 4 \\
\hline - inflata, Chemn. (Pecten) & & . & 3 & & 2 & 33 & & & & 2 & 3 & 3 \\
\hline - Loscombi? Sow. . . . & & . & & & & 2 & & & & & 3 & 3 \\
\hline - peregrina, May. . . . & & . & & & 2 & & & & & & & \\
\hline
\end{tabular}


Lima squamosa. Lam.

Perna Soldanii, Desh.

Avicula (Meleagrina) Studeri, May.

- ? peregrina, May.. . . . .

Pina Bachmanni? May. . . . . . . . . .

- Brocchii, Orb. . . . . . . 4

Brocchii, Or

- tetragona, Broc.

Mytilas Aquitanieus, May.

- oblitus, Mich. . . . . . .

- (Modiola) barbatus, L. . . .

- $\quad$ - $\quad$ costulatus, Risso. .

-- $\quad$ - Dolfusi, May. . .

- $\quad$ Studeri, May.. . .

- cinnamomcus, Chemn. (Myt.)

-- Tithophagus, L. (Mytilus)

Congeria Basteroti, Desh. (Mytilus)

Arca barbata, L. . . . . . . . .

- Breislacki, Bast. . . . . . .

- clathrata, Defr. . . . . . . .

- diluvii, Lam. . . . . . . .

- Fichtcli, Desh. . . . . . .

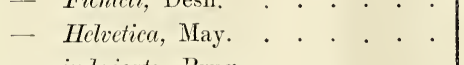

- imbricata, Brug. . . . . . . . 3

- lactca, L. . . . . . . . . . .

- latisulcata? Nyst. . . . . . . 3

— Noe, L. . . .

- Okeni, May. • . • . . . .

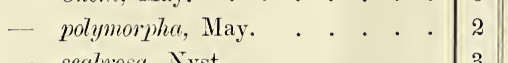

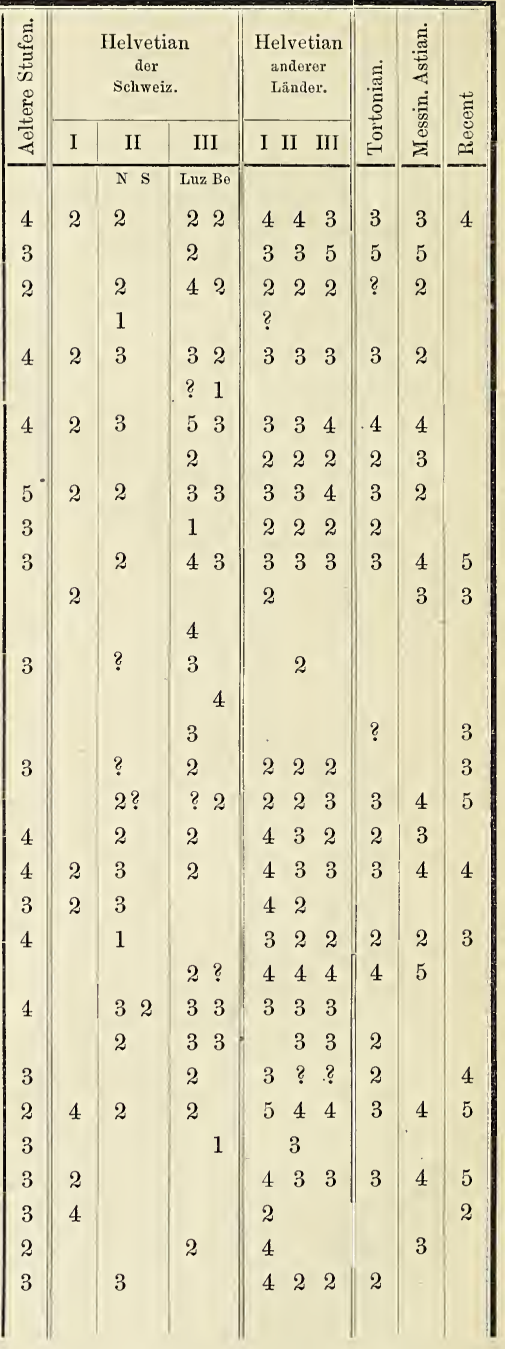




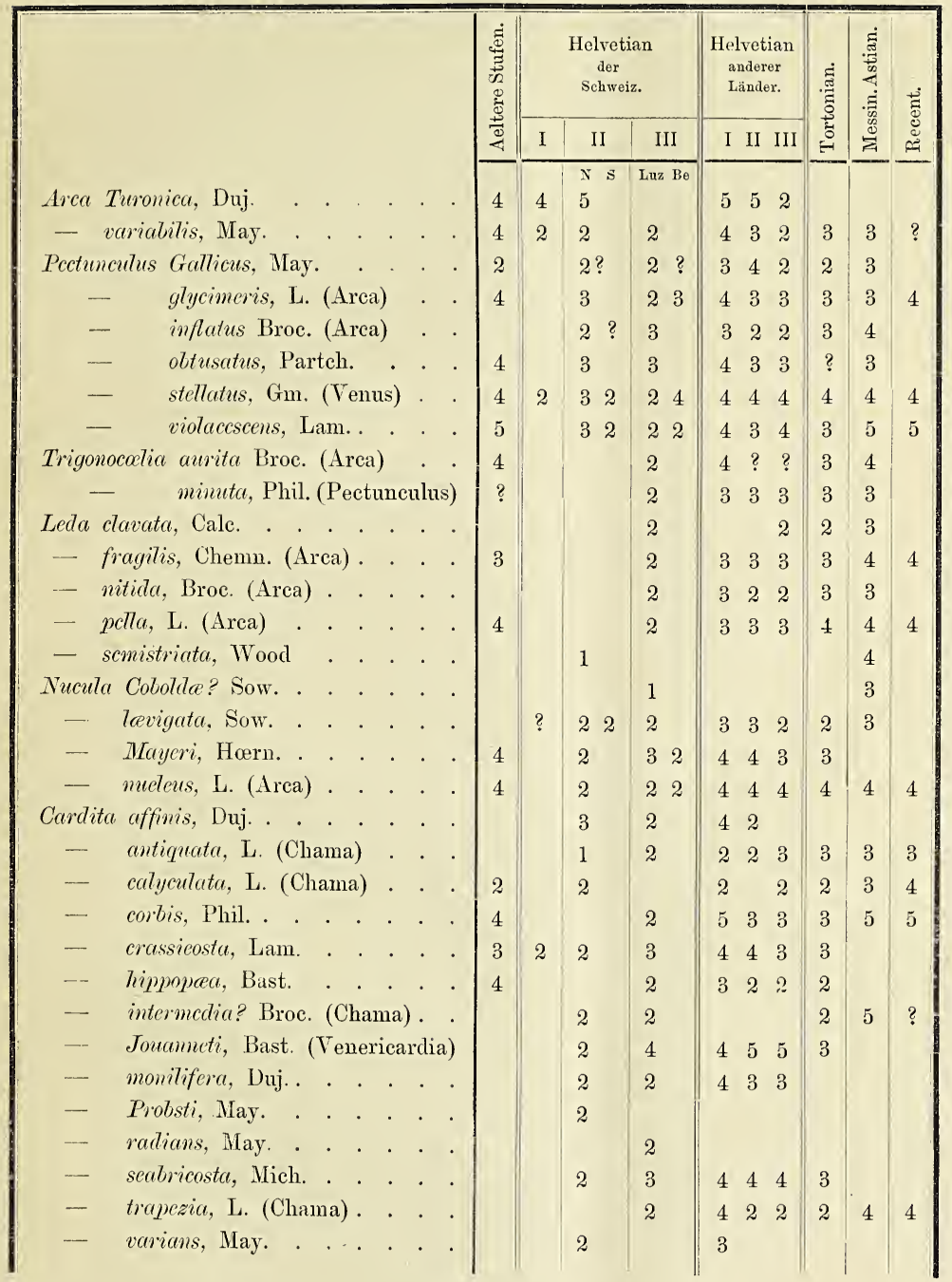




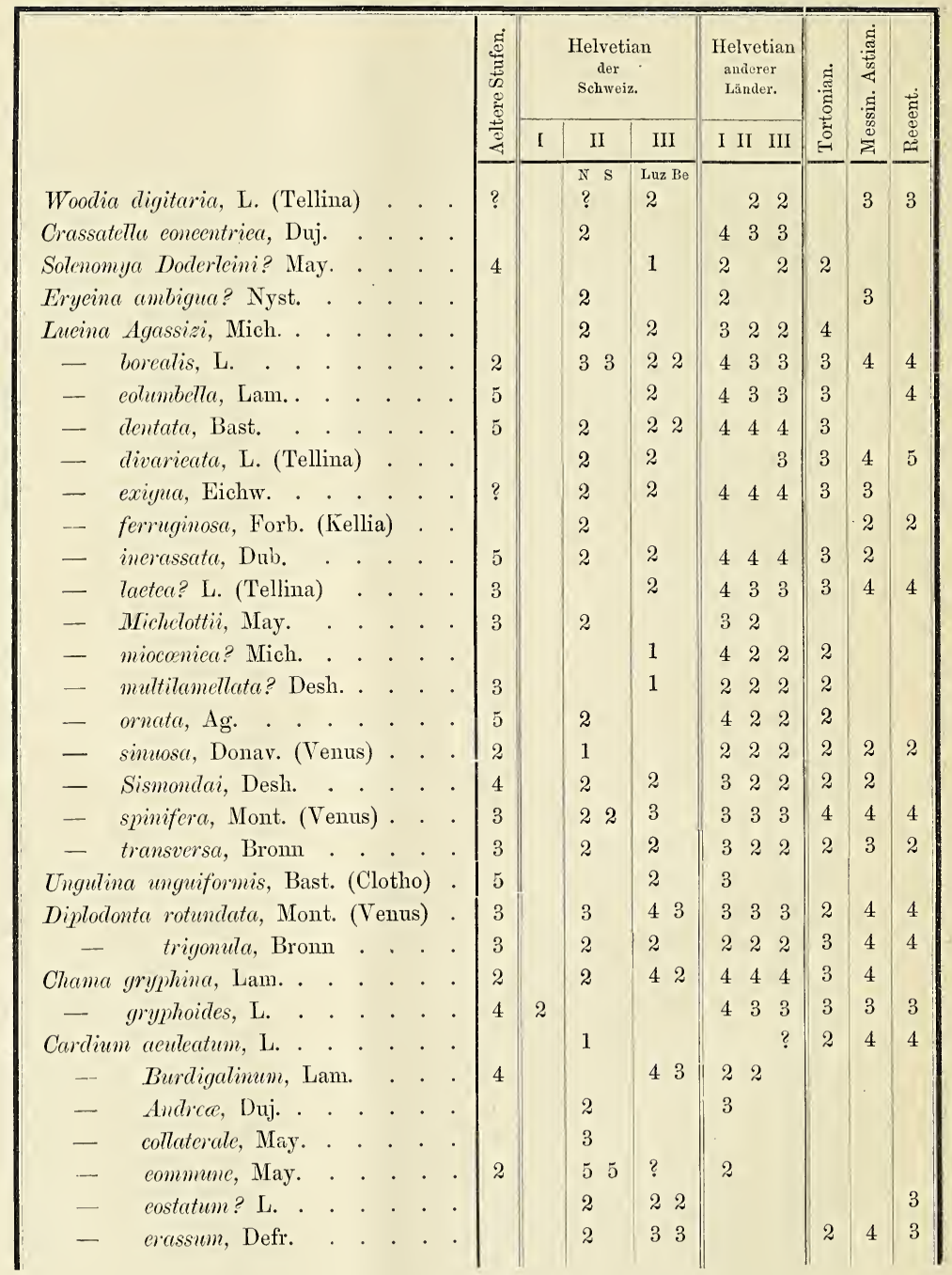




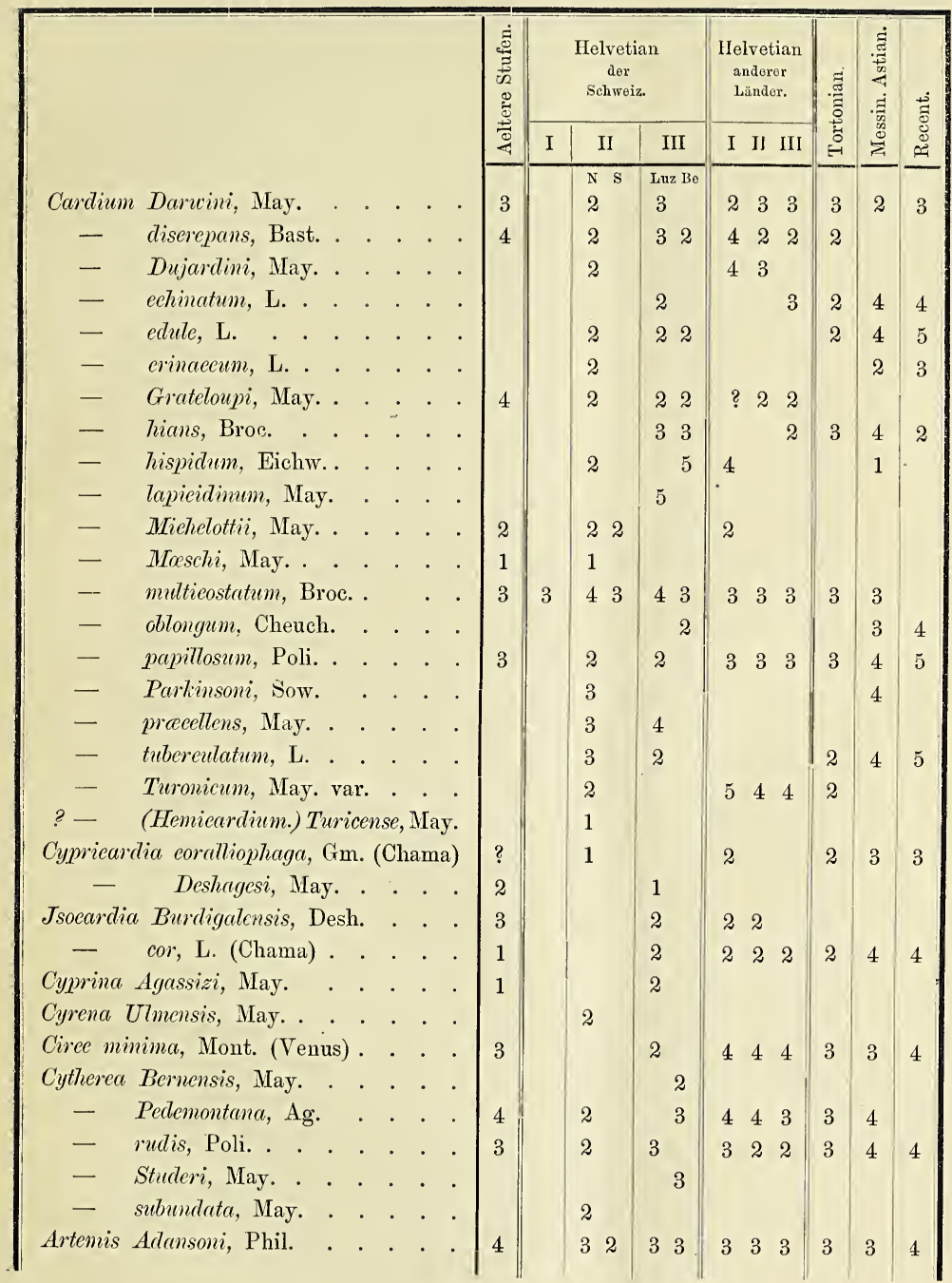


Artemis exoleta, L. (Venus)

- lincta, Penn. (Venus)

- orbicularis, Ag.

Lucinopsis Lajonkairei, Payr. (Venerupis)

Venus Basteroti, Desh. . . . . . .

- Brocchii, Desh.

- casina? L. .

- clathrata, Duj.

- coturnix, Duj.

- fasciculata, Reuss.

- Haidingeri, Hœrn.

- multilamella, Lam. (Cytherea)

- ovata, Paun. . . . . . . .

- plicata, Gm.

- rusticula, May.

- umbonaria, Lam. (Cyprina).

- verrucosa, L. . . . . . . .

- Vindolonensis, May. . . . 2

Tapes Basteroti? May. . . . . . . 4

- clandestina? May. . . . . . 3

- Helvetica, May.

- - var. crassissima, May.

- Montispeleensis, May. . . . .

- puella? May. . . . . . . .

- Ulmensis, May.

- vetula, Bast. (Venus)

Petricola lithophaga, Retz. (Venus) . .

Donax lucidus, Eichw.

- transversus, Desh. . . . . . 5

Psammobia incarnata, Penn. . . . . 3

- Labordei, Bast. (Soletellina)

- vespertina? L. (Tellina) .

Strigilla camaria, L. ('Tellina) . . .

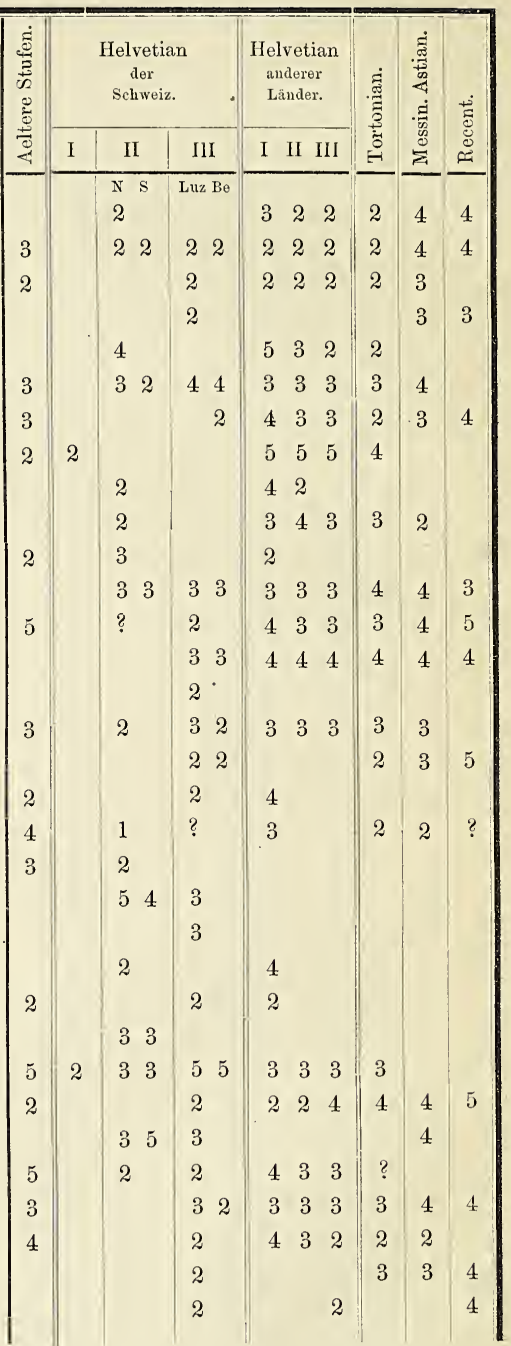




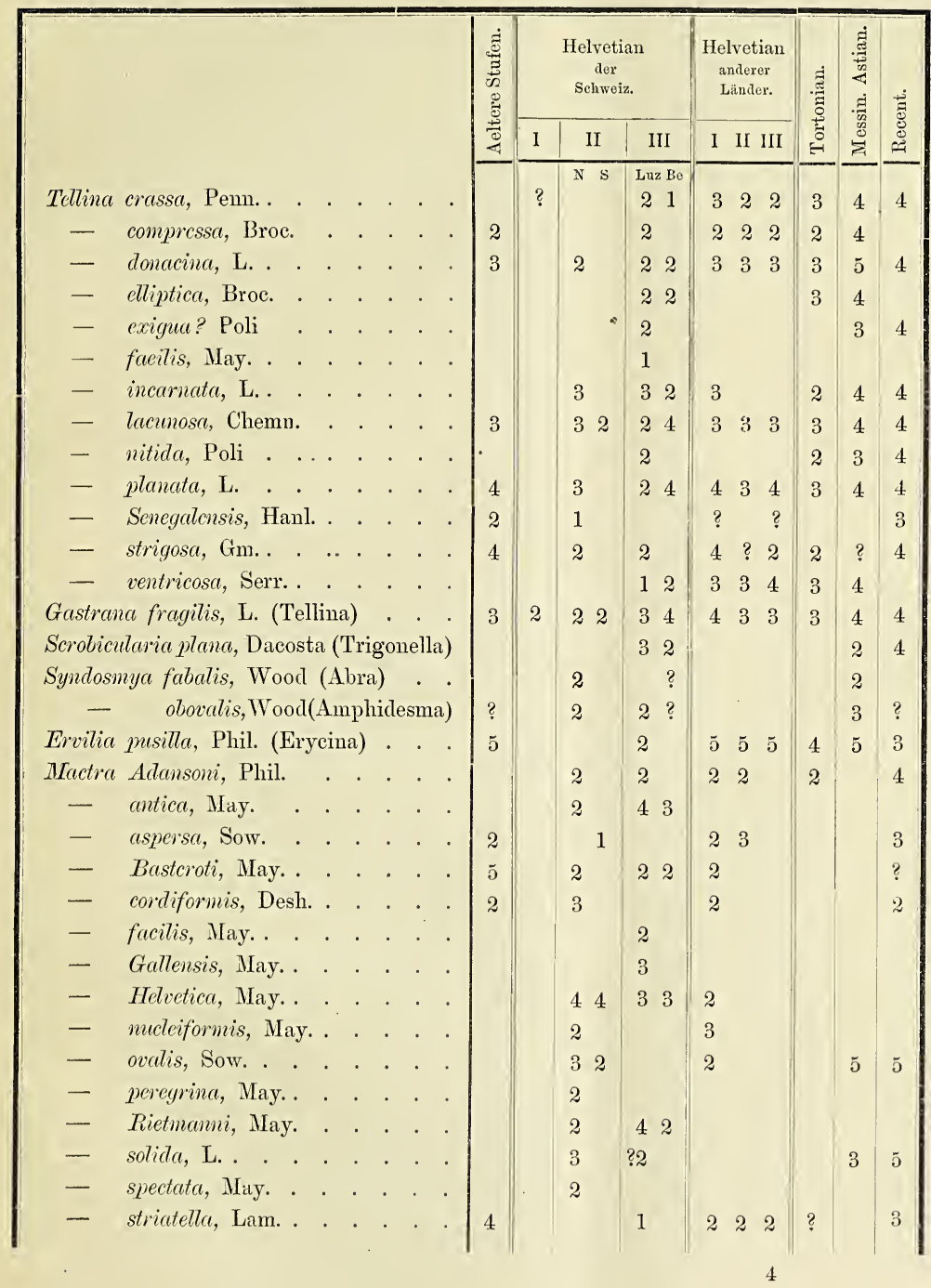




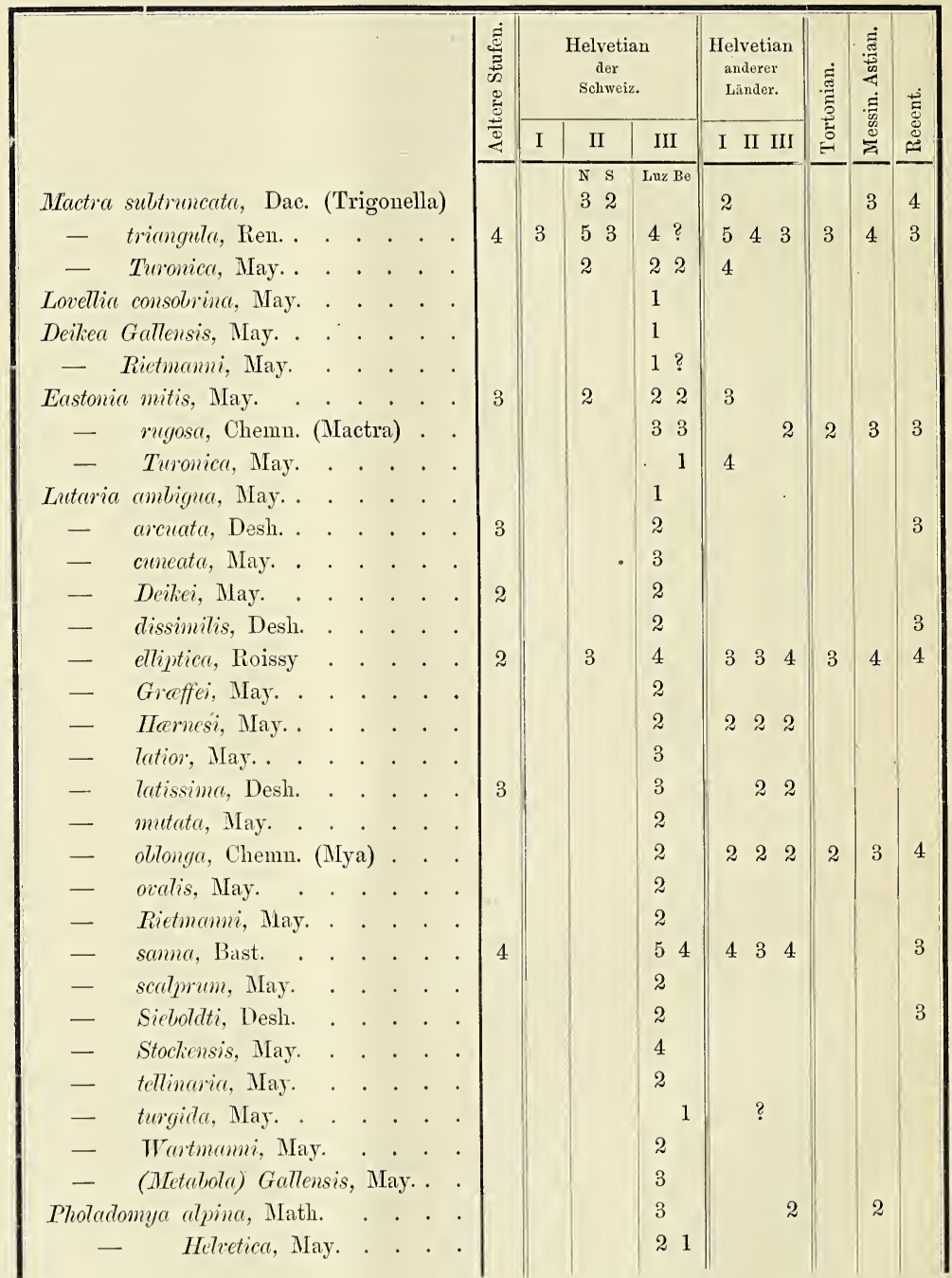




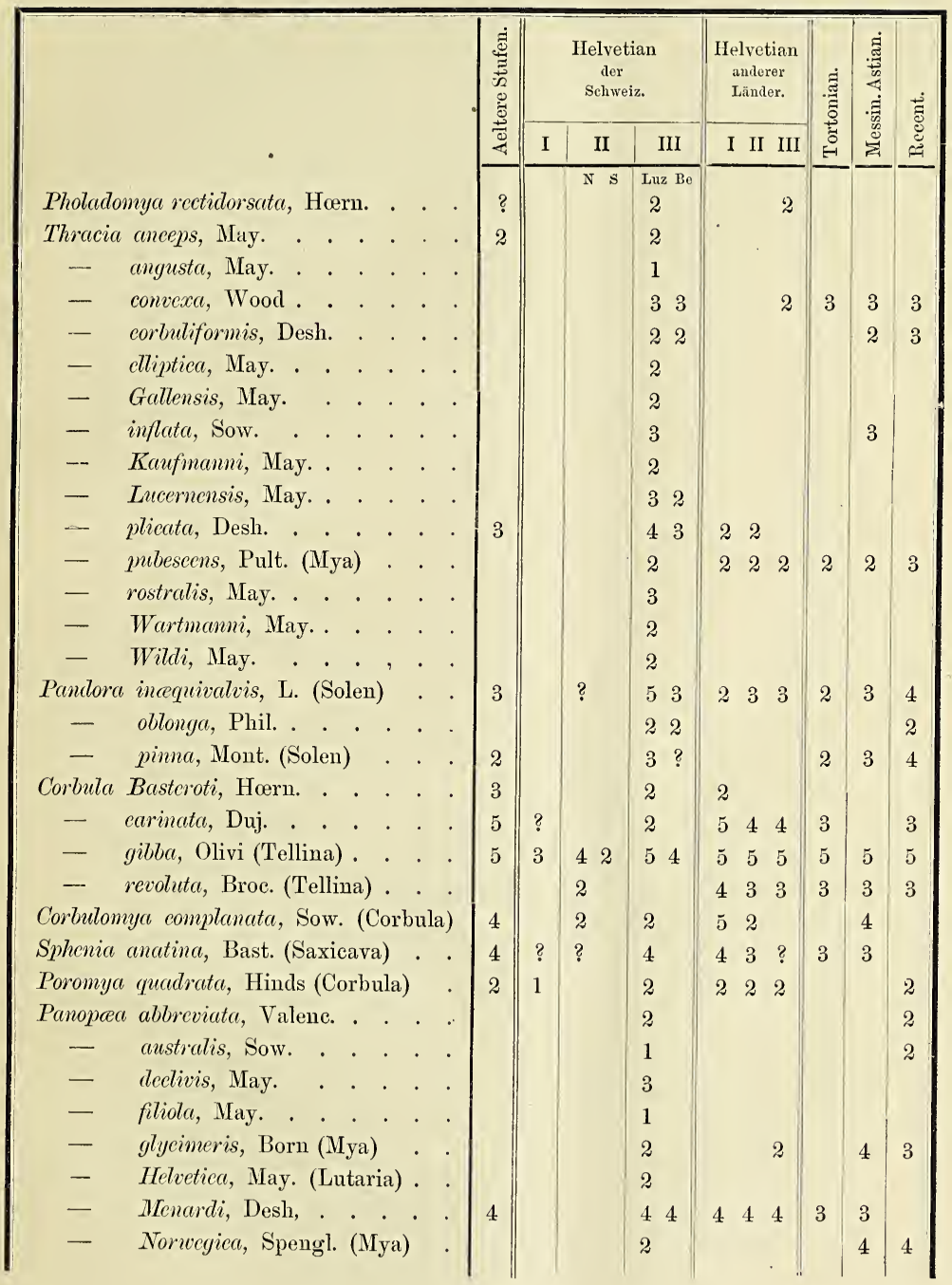




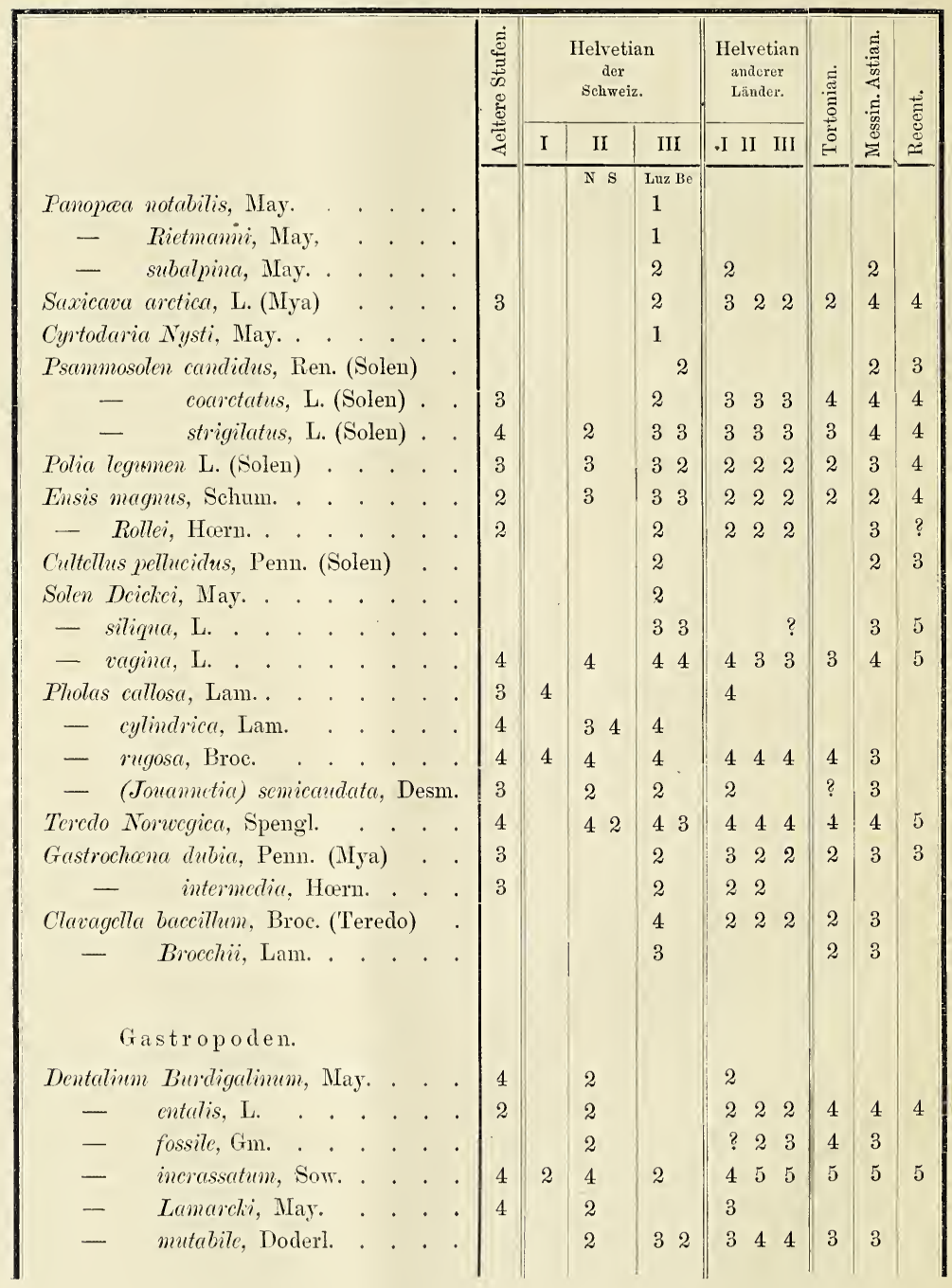


Dentalium sexangulare? Gim. . . . .

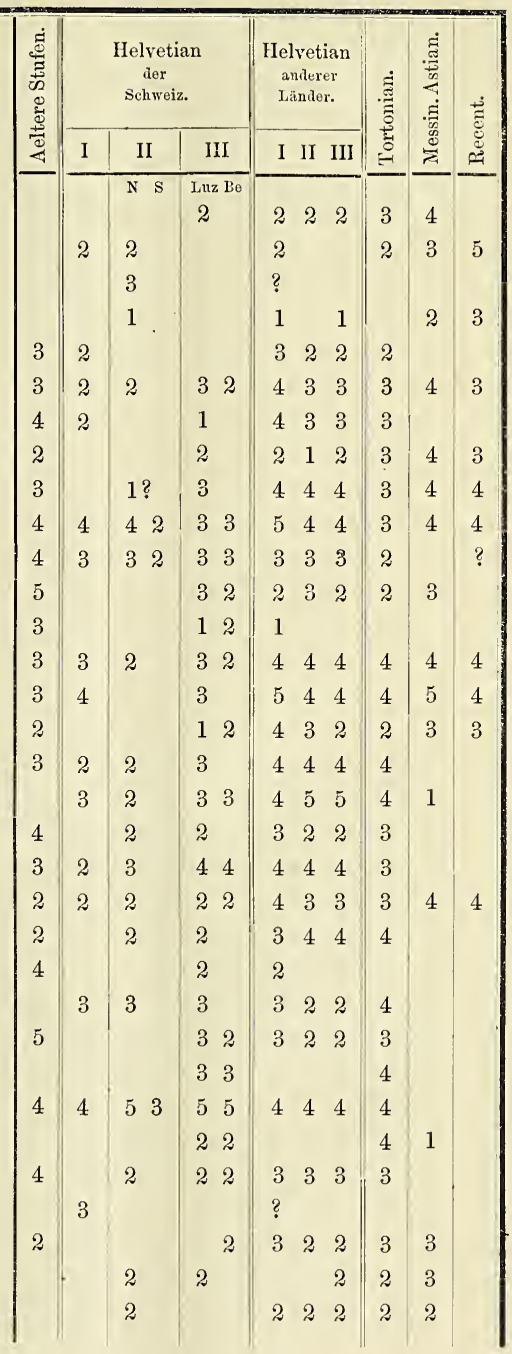


Scalaria (Doderleinia) lanceolata, Broc. (Turbo) .

Scalaria (Doderleinia) torulosa, Broc. (Turbo)

Mathilda quadricarinata, Broc. (Turbo) Litorina famulum, May.

- ? Pillintoni, May. . . .

Rissoa curta, Duj. . . . . . . . 3

Fossarus costatus, Broc. (Nerita) . .

Adeorlis planortillus, Duj. (Solarium) . - subcarinatus, Brown (Trochus)

Melania Escheri, Brongn. . . . . .

Melanopsis Aquensis? Grat. . . . .

$$
\text { - } \quad \text { citharella, Merian . . . . }
$$

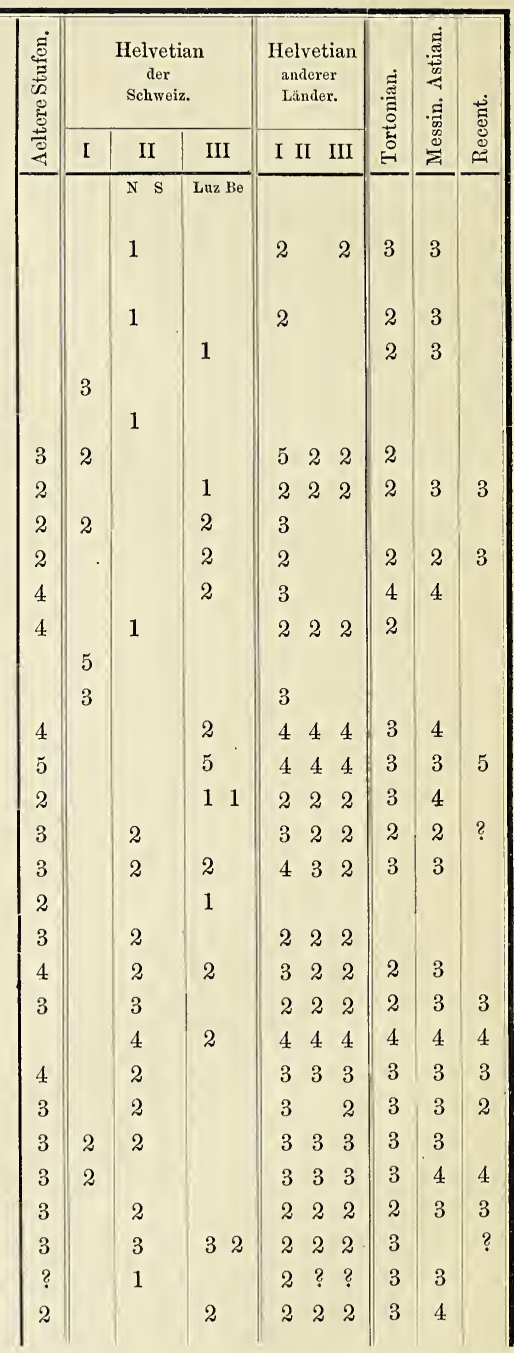

Bithynia acuta, Drap. (Cyclostoma).

Niso elurnea, Risso . . . . . . . 2

Eulima lactea, Grat. (Melania) . . . 3

Pyramidella unisulcata, Duj. . . . . 3

Tornatella papyracea? Bast. . . . . 2

- pinguis, Orb. . . . . . 3

- semistriata, Fér. . . . . 4

- tornatilis L. (Helix) . . . 3

Auriculina buccinea, Broc. (Voluta)

- stivata, Phil. . . . . . 4

Bulla Brocchii? Mich. . . . . . . 3

- convoluta, Broc. . . . . . .

- lignaria, L. . . . . . . .

- utricula, Broc. . . . . . . 3

Solarium carocollatum, Lam. . . . . 3

- millegramum, Lam. . : . .

- simplex, Bronn. . . . . . 


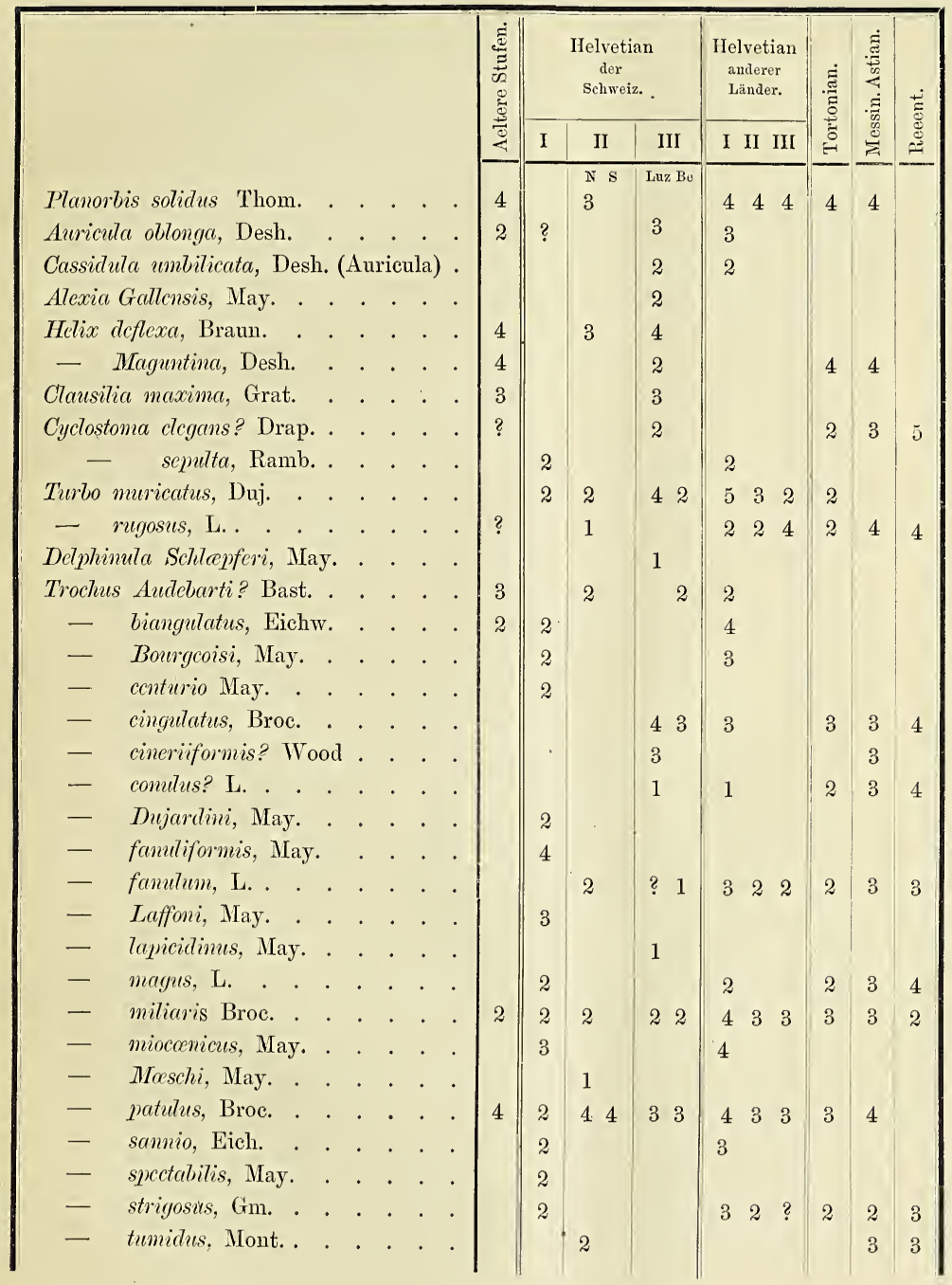




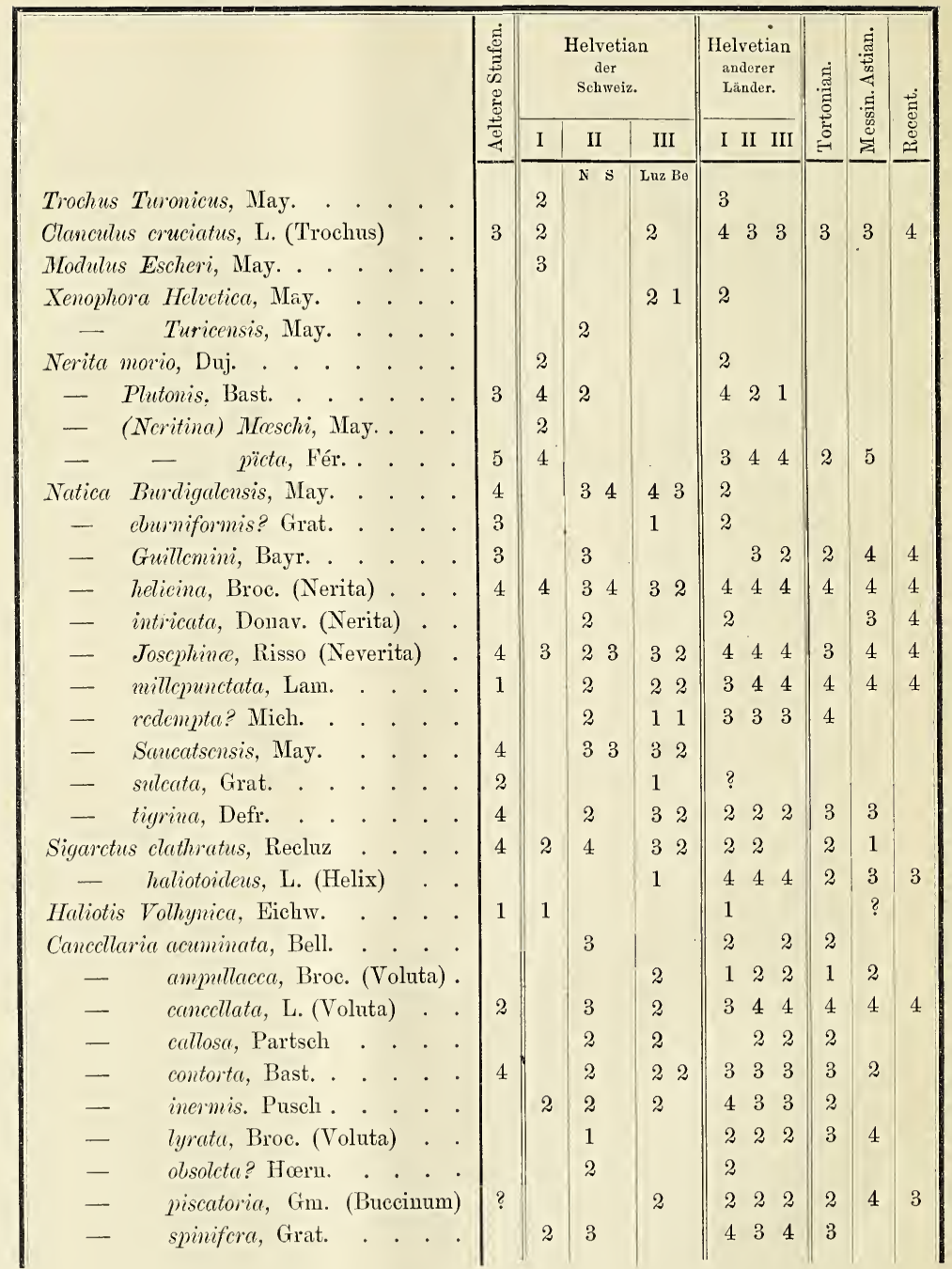




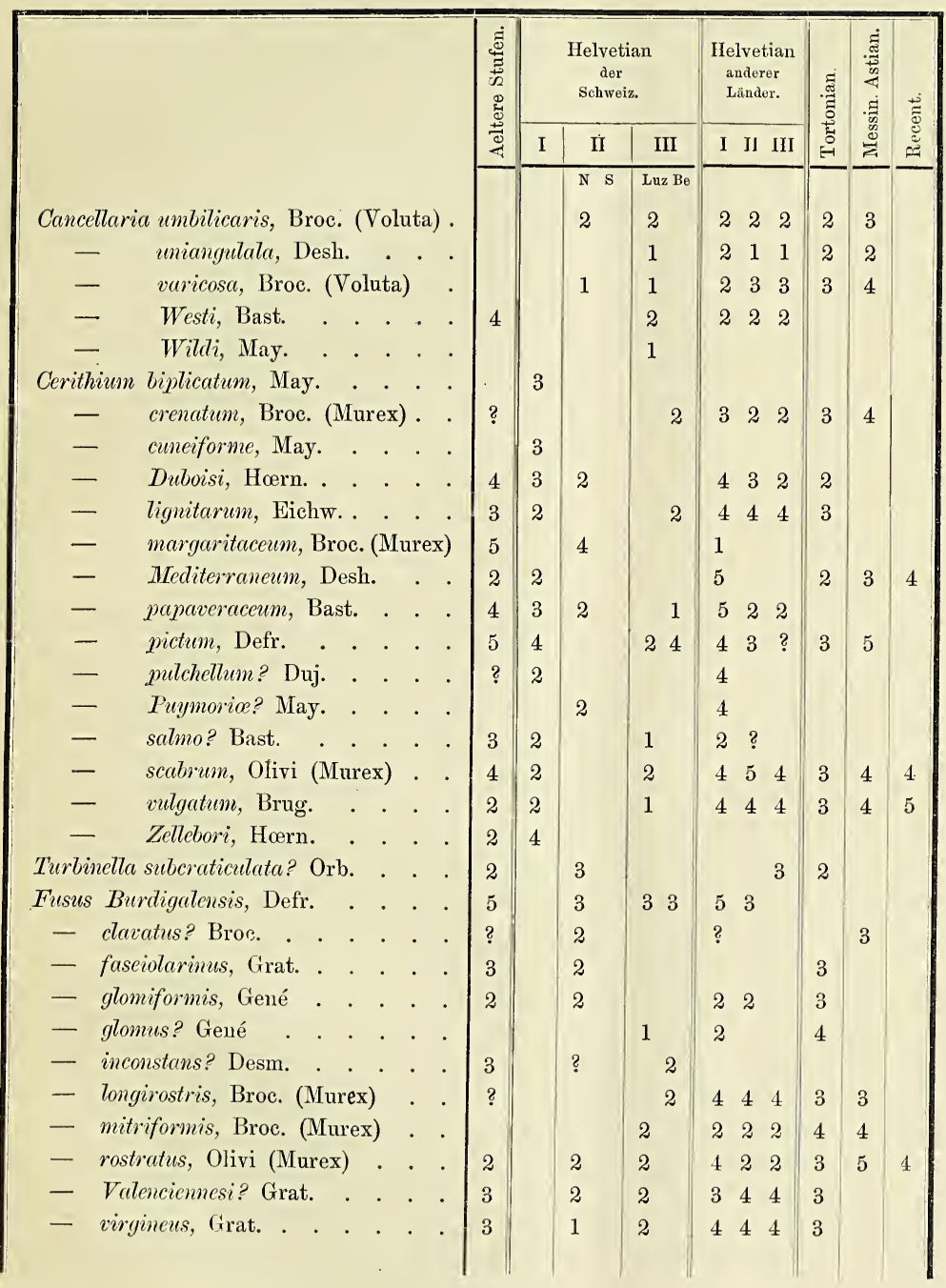




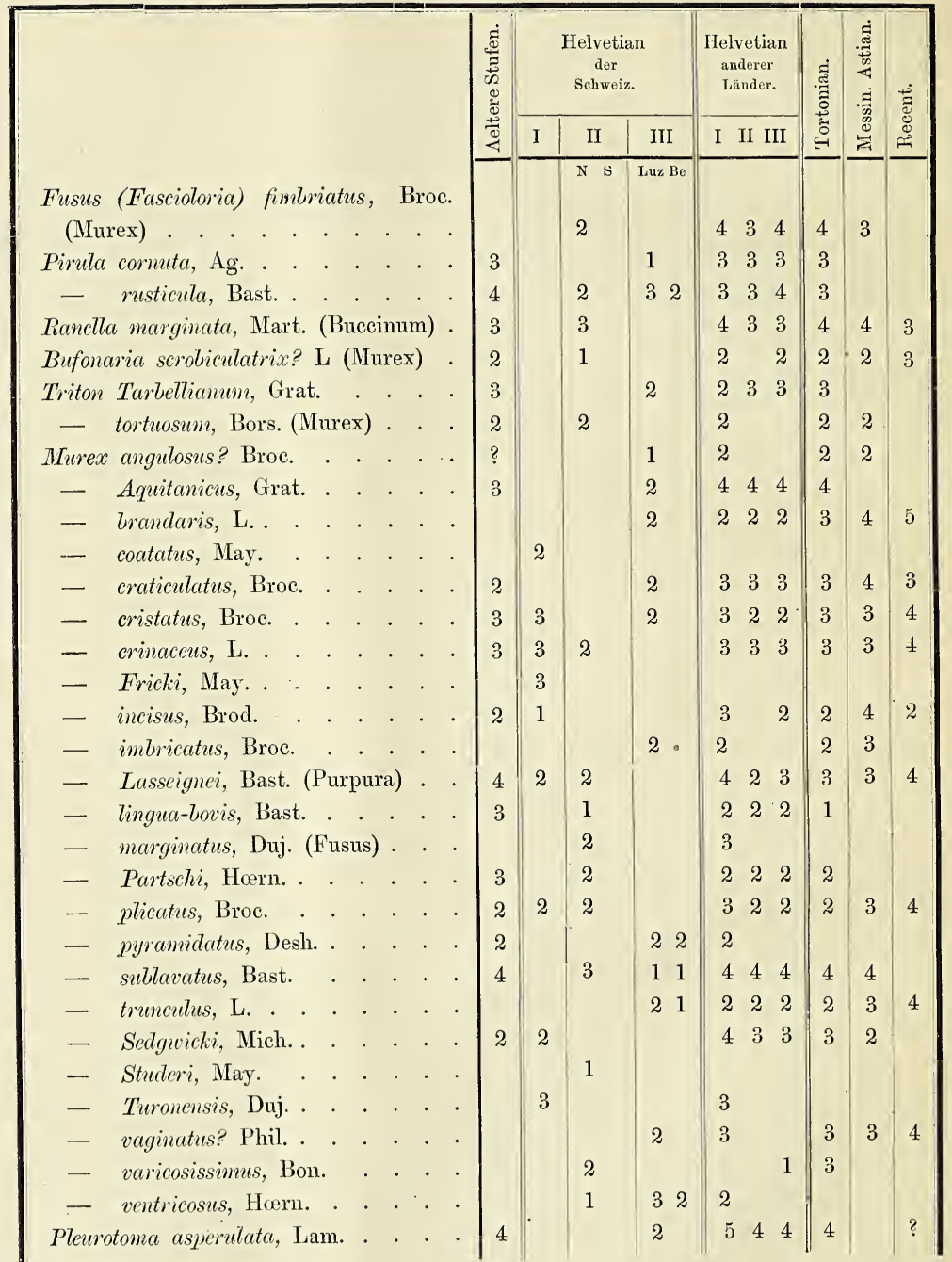




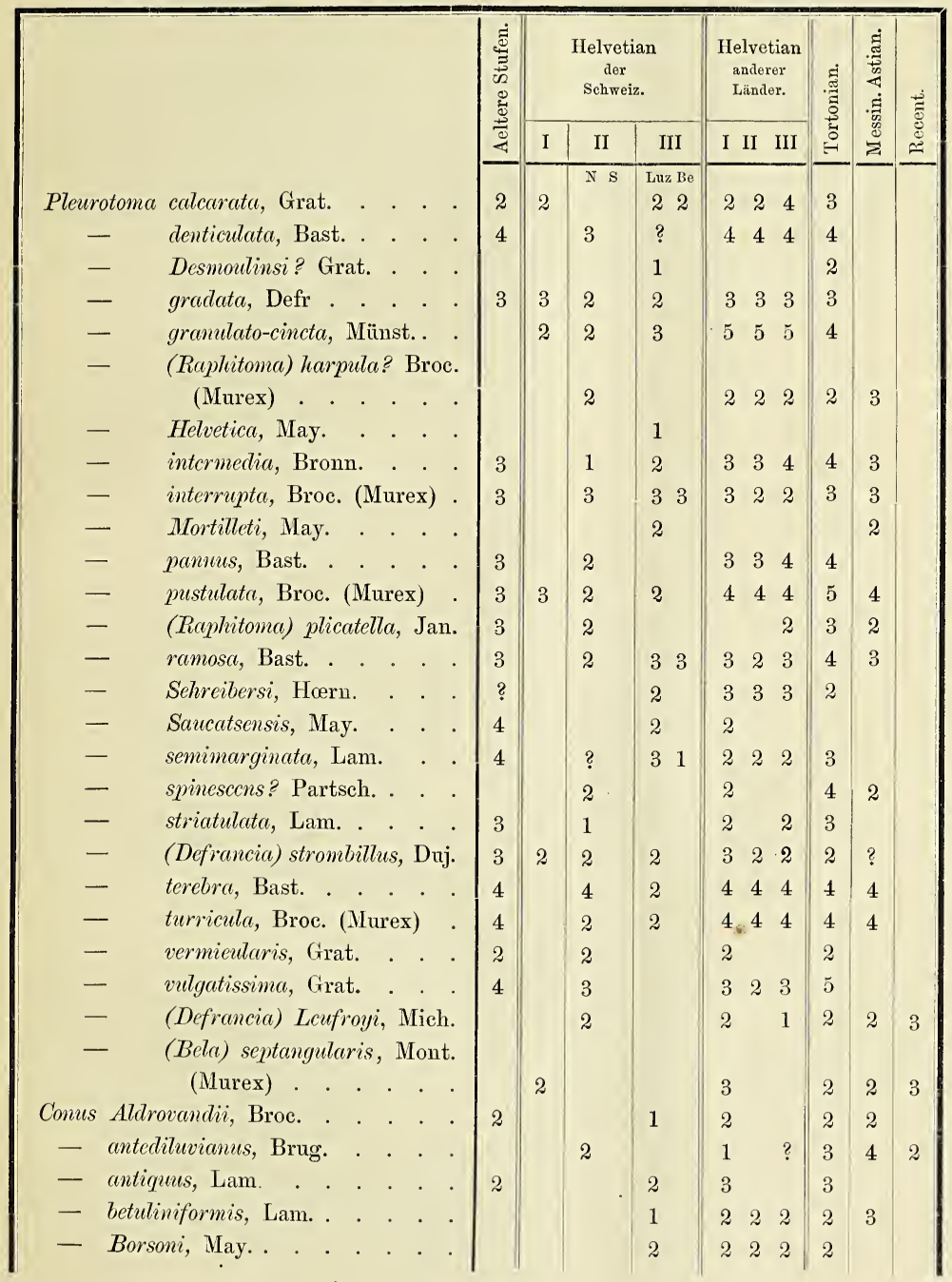




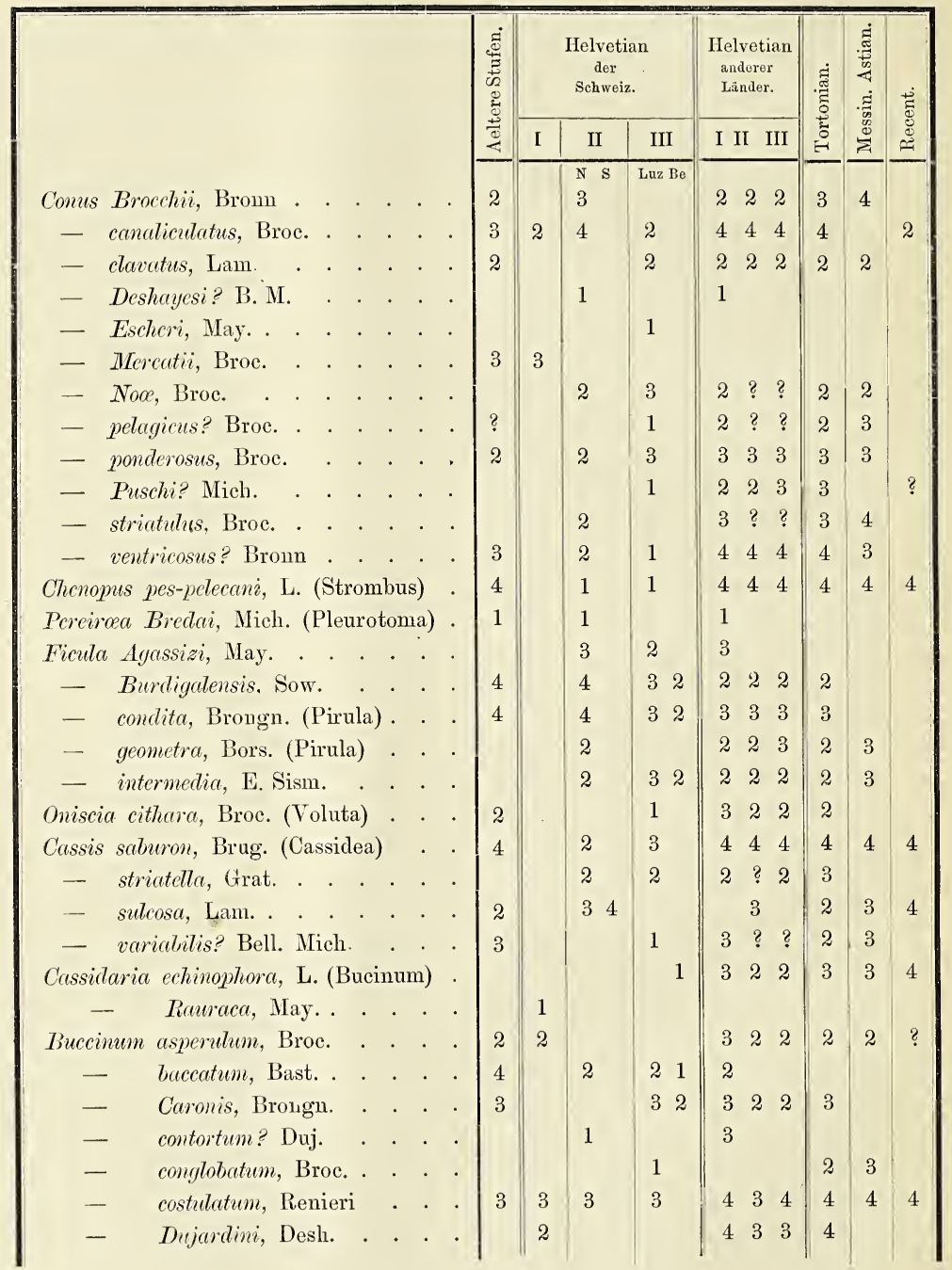




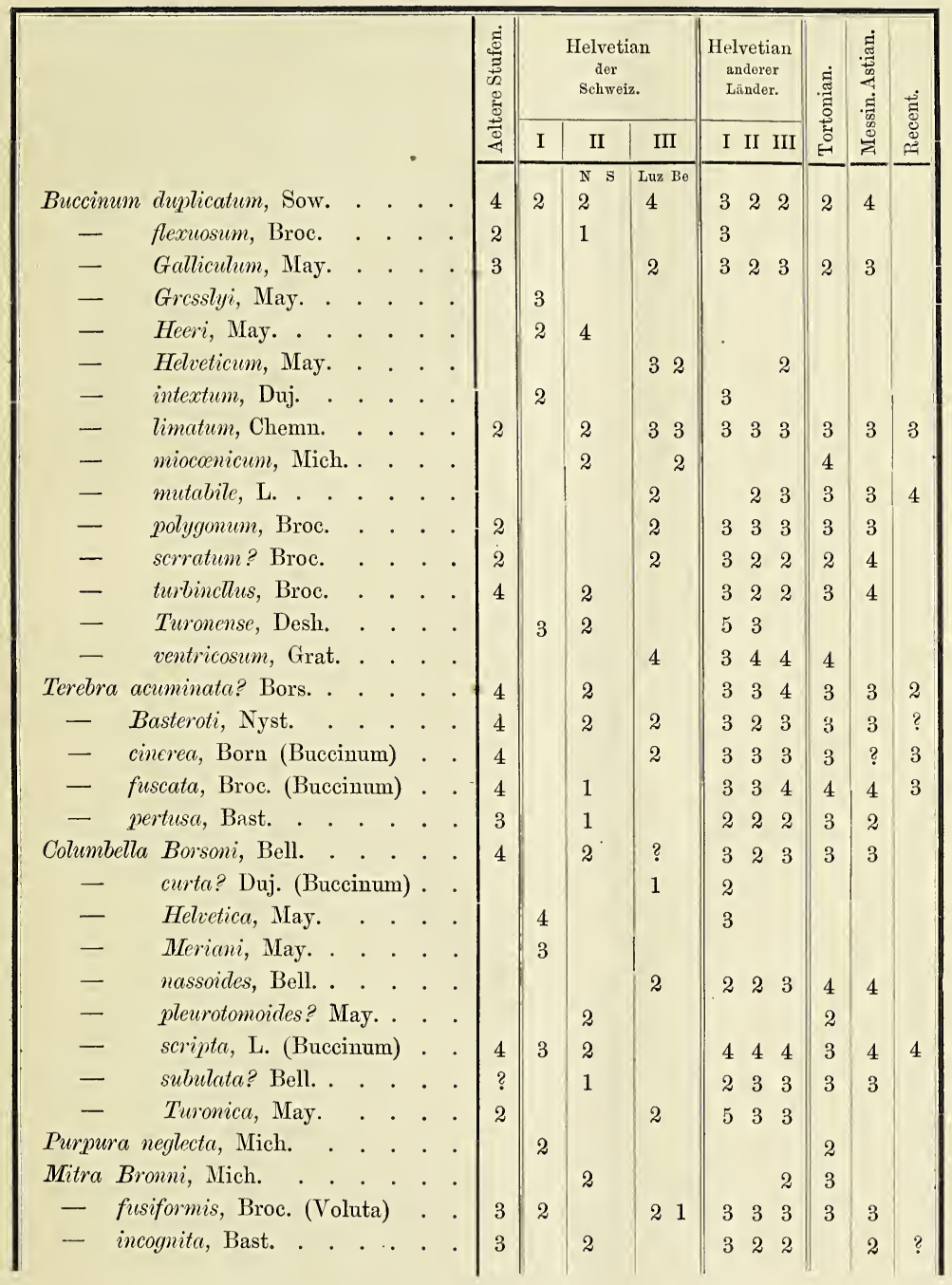


Mitra scrobieulatu, Broc. (Voluta). . - striatula, Broc. (Voluta) . .

Marginella miliacea, Lam. . . . . . . 2

Voluta? Bernensis, May. . . . . .

Volutara rispina, Lam. . . . . . . 4

Erato locvis, Sow. . . . . . . . . 3

Ovula spelta, Lam. . . . . . . . 2

Cyproe amygdalum, Broc. . . . . 2

- elonguta, Broc. . . . . . .

- fabagina, Lam. . . . . . . 3

- pirum, Gm. . . . . . . . 2

Ancillaria glandiformis, Lam. . . . 4

Oliva elavula, Lam. . . . . . . . 4

- flammulata, Lam. . . . . . . 4

Megasiphonia Aturi, Bast. (Nautilus) .

Cyrrhopoden.

Balanus Holgeri, Gein.

- oblique-striatus, Fischer

- palmatus, Lam.

- sulcatus, Brug.

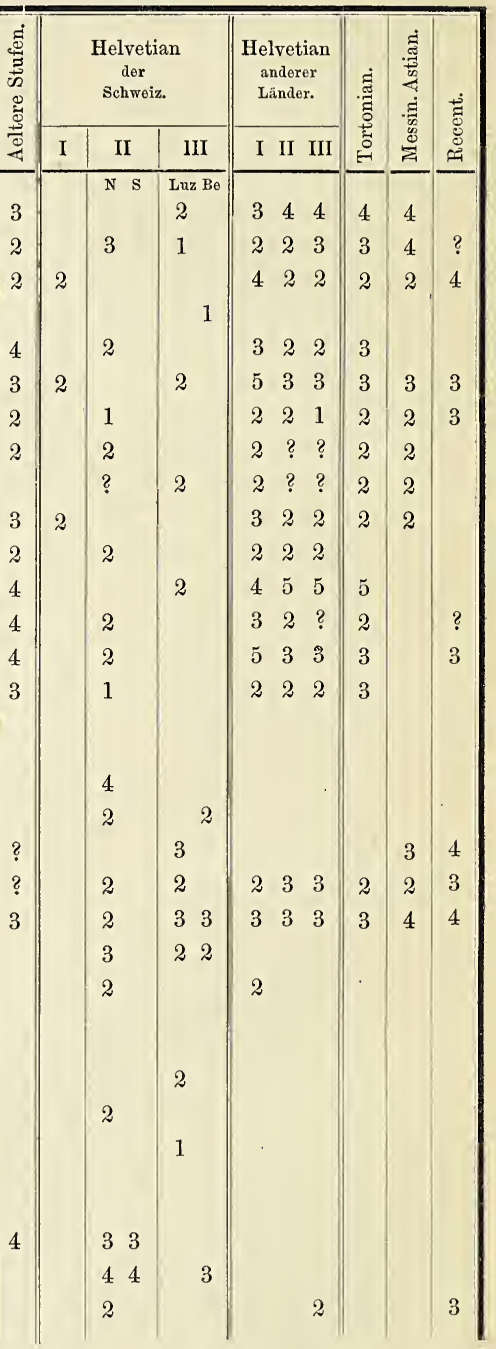

- tintimabulum, L. . . . . . . 3

- undulatus, Fischer . . .

Pyrgoma sororeula? May. . . . . .

Crustaceen.

Caneer Rietmanni, May.

Lupea? dubia, May. .

Astaeus? Lucernensis, Mav.

W ï rmer.

Serpula comugata? Goldf. . . . . . 4

- cremulosa? May.

- ammlata? Lam.

2 


\begin{tabular}{|c|c|c|c|c|c|c|c|c|c|c|c|c|c|c|}
\hline \multirow[b]{3}{*}{ F ische. } & & & & & 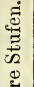 & & $\begin{array}{l}\text { Helvet } \\
\text { der } \\
\text { Schive }\end{array}$ & & & & $\begin{array}{l}\text { etian } \\
\text { rer } \\
\text { der. }\end{array}$ & 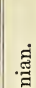 & 密 & \\
\hline & & & & & & $\mathrm{I}$ & II & III & & & I III & $\overbrace{0}^{0}$ & $\sum_{2}^{2}$ & \\
\hline & & & & & & & $\mathrm{N} \mathrm{s}$ & Luz Be & & & & & & \\
\hline Lamna contortidens, Ag. & . & . & . & & 2 & & 33 & 2 & & & 32 & & & \\
\hline - cuspidata, Ag. . . & . & . & . & . & & & 43 & 22 & & 2 & 42 & & & \\
\hline - denticulata, Ag. . & 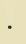 & . & . & & & & 3 & & & & & & & \\
\hline - dubia, Ag. . . . & . & . & . & & & & 42 & & & & 4 & & & \\
\hline - elegans, Ag. . . . & . & . & . & . & 3 & & 2 & & & 2 & & & & \\
\hline Oxyrhina Desori, Ag. . . & 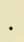 & . & . & . & & & 32 & & & 2 & 33 & & & \\
\hline - hastalis, Ag. . . & . & . & . & . & & & 43 & 2 & & & 22 & & & \\
\hline - leptodon, Ag. . . & . & . & . & & & & 2 & & & & & & & \\
\hline Carcharodon Helveticus, May. & . & - & . & . & & & 2 & & & & 2 & & & \\
\hline megalodon, Ag. & 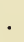 & . & . & . & 2 & & 2 & & & & 3 & 2 & ? & \\
\hline microdon, Ag. & $\cdot$ & 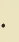 & . & & & & 2 & & & & & & & \\
\hline polygyrus, Ag. & . & . & . & . & 2 & & 32 & $2 ?$ & & & 22 & & & \\
\hline Turicensis, May. & & & . & & & & 1 & & & & & & & \\
\hline - turgidus, Ag. . & . & . & . & & 2 & & 2 & & & & & & & \\
\hline Hemipristis serra, Ag. . . & . & • & . & & 2 & & 32 & 2 & & & 22 & & ? & \\
\hline Galeocerdo aduncus, Ag. . & & & . & & 2 & & 32 & & & & & & & \\
\hline - latidens, Ag. & • & & . & & & & 2 & & & & 2 & & & \\
\hline - minor, Ag. . . & & & . & & 2 & & 2 & & & & & & ? & \\
\hline Notidanus primigenius, Ag. & - & • & . & & 2 & & 22 & 2 & & & & & & \\
\hline Zygobatis Studeri, Ag. . . & & & . & & & & 32 & & & & & & & \\
\hline Myliobatis Stembergi? Ag. & & & - & & & & 2 & & & & & & & \\
\hline Aetobatis arcuatus, Ag. . & & & . & & & & 2 & & & & & & & \\
\hline Sparoides Quenstedti, May. & & & 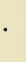 & & & & 3 & 22 & & & & & & \\
\hline Cetaceen. & & & & & & & & & & & & & & \\
\hline Halitherium Schinzi, Kaup. & & & & & & & 2 & & & & & & & \\
\hline Hatianassa Studeri, Mey.. & & & . & & ? & & 3 & & & & 3 & & & \\
\hline
\end{tabular}






